\title{
Delay-active damage versus non-local enhancement for anisotropic damage dynamics computations with alternated loading
}

\author{
R.Desmorat ${ }^{\mathrm{a}}$, M. Chambart ${ }^{\mathrm{a}}$, F. Gatuingt ${ }^{\mathrm{a}}$, D. Guilbaud ${ }^{\mathrm{b}}$ \\ ${ }^{a}$ LMT-Cachan (ENS Cachan/CNRS/UPMC/PRES UniverSud Paris) \\ 61, avenue du Président Wilson, F-94235 CACHAN CEDEX, France \\ ${ }^{b} C E A, D E N, D M 2 S$, SEMT, Laboratoire d'études dynamiques \\ F-91191 GIF-SUR-YVETTE
}

\begin{abstract}
Anisotropic damage thermodynamics framework allows to model the concrete-like materials behavior and in particular their dissymmetric tension/compression response. To deal with dynamics applications such as impact, it is furthermore necessary to take into account the strain rate effect observed experimentally. This is done in the present work by means of anisotropic visco-damage, by introducing a material strain rate effect in the cases of positive hydrostatic stresses only. The proposed delay-damage law assumes no viscous effect in compression as the consideration of inertia effects proves sufficient to model the apparent material strength increase. High-rate dynamics applications imply to deal with wave propagation and reflection which can generate alternated loading in the impacted structure. In order to do so, the key concept of active damage is defined and introduced within both the damage criterion and the delay-damage evolution law.

At the structural level, strain localization often leads to spurious mesh dependency. Three-dimensional Finite Element computations of dynamic tensile tests by spalling are presented, with visco-damage and either without or with non-local enhancement. Delay-damage, as introduced, regularizes the solution in fast dynamics. The location of the macrocrack initiated is found influenced by non-local regularization. The strain rate range in which each enhancement, delay-damage or non-local enhancement, has a regularizing effect is studied.
\end{abstract}

Key words: anisotropic damage, concrete, non-local, visco-damage, micro-cracks closure

Email address: desmorat@lmt.ens-cachan.fr (R.Desmorat) 


\section{Nomenclature}

\begin{tabular}{|r|l|}
\hline \hline Notation & Meanning \\
\hline \hline$\langle x\rangle_{+}=\max (x, 0)$ & positive part of scalar $x$ \\
$\langle x\rangle_{-}=\min (x, 0)$ & negative part of scalar $x$ \\
$\langle\boldsymbol{T}\rangle_{+}$ & positive part of symmetric second order tensor $\boldsymbol{T}$ \\
$\boldsymbol{X}: \boldsymbol{Y}, \mathbb{X}$ & contracted product $X_{i j} Y_{i j}, X_{i j k l} Y_{k l}$ \\
$\boldsymbol{1}$ & Heaviside function, $\mathcal{H}(x)=1$ if $x \geq 0, \mathcal{H}(x)=0$ if $x<0$ \\
$\operatorname{tr} \boldsymbol{T}$ & second order unit tensor \\
$\boldsymbol{T}^{D}=\boldsymbol{T}-\frac{1}{3} \operatorname{tr} \boldsymbol{T} \mathbf{1}$ & deviatoric part of second order tensor $\boldsymbol{T}$ \\
\hline$A, a$ & damage parameters \\
$\dot{D}_{\infty}$ & delay-damage parameters \\
$d_{a c t}$ & active damage \\
$\boldsymbol{D}$ & second order damage tensor \\
$E, v$ & elasticity parameters \\
$\mathbb{E}$ & Hooke's tensor \\
$f$ & damage criterion function \\
$l_{c}$ & characteristic length \\
$\hat{\varepsilon}$ & Mazars equivalent strain \\
$\hat{\varepsilon}^{n l}$ & non local Mazars equivalent strain \\
$\boldsymbol{\varepsilon}$ & strain tensor \\
$\kappa_{0}$ & damage threshold \\
$\dot{\lambda}$ & damage multiplier \\
$\rho$ & density \\
$\boldsymbol{\sigma}$ & stress tensor \\
$\tilde{\boldsymbol{\sigma}}$ & effective stress tensor \\
$\boldsymbol{\sigma}^{D}$ & deviatoric stress tensor \\
$\psi$ & Helmholtz specific free energy \\
$\psi^{\star}$ & Gibbs specific free enthalpy \\
& \\
\hline
\end{tabular}

\section{Introduction}

The degradation mechanisms of quasi-brittle materials such as concrete are mainly governed by the nucleation and the propagation of micro-cracks. Present within the representative volume element (RVE) of Continuum Mechanics, these micro-cracks lead to strain softening and degrade the elastic properties. When unloading, the micro-cracks partially close, some permanent strains remain and partial stiffness recovery is observed [1,2].

Concrete behavior is therefore traditionally modeled by use of plasticity and/or damage constitutive equations. Plasticity models the permanent strains and the softening behavior if a negative hardening is considered $[3,4,5,6]$ but the degradation of elasticity parameters is not represented. Theories of elasto-(visco-)plasticity coupled with damage $[7,8,9,10,11,12,13,14]$ can be considered for general loading conditions. They lead to numerical complexity, often for quasi-brittle materials because of the introduction of several criterion functions written in different spaces (strains space for damage and stresses space for plasticity) and they involve a large number of material parameters. For simplicity and efficiency, a possibility (valid for not too high confinement) is to consider elasticity coupled with damage only. This continuous damage framework, classical for concrete-like materials [15], is the one considered here.

Tensorial damage has to be introduced when the observed micro-cracking pattern is anisotropic [16, 17, 18, 19, $20,21,22,23,24,25,26]$. This leads to mathematical complexity. But taking into account the induced anisotropy (i.e. to put more physics in the model) allows to reduce the numbers of material parameters [27, 28]. This also allows to propose damage models fully consistent with thermodynamics of solids materials [11, 13, 29]: a single (state) damage variable represents any micro-cracking state, either due to tension, to compression or to any $3 \mathrm{D}$ tensile or compressive loading. 
The dynamics behavior of quasi-brittle materials has been modeled within Continuum Damage Mechanics framework [30]. The relative increase of concrete apparent strength with the loading rate (or strain rate effect [31, 32, 33]) is gained by introducing viscous effects in so-called visco-damage or delay-damage laws [8, 37, 38, 39, 40, 41, 42, 43]. Most of the damage models which do not consider plasticity - i.e. pure visco-damage models - either give a symmetric tension/compression response, or consider an isotropic damage represented through a tensile damage variable $D^{t}$ and a compressive one $D^{c}$. As mentioned above, this feature is not consistent with the thermodynamics definition of a state variable representing a micro-cracking pattern (whatever the loading sign), but it is quite efficient to make uncoupled (visco-)damage evolutions in tension and in compression (and also to make uncoupled the strain rate effects in tension and in compression). Doing so, two criterion functions and two viscosity laws have to be considered (each time one for tension, one for compression), increasing the number of material parameters to identify [38, 41].

Dissymmetry tension/compression in quasi-static monotonic loading can now - in a not too complex manner be modeled by means of induced anisotropic damage. The case of alternated loading is quite more difficult to handle and has not been studied much in quasi-static or slow - such as seismic - dynamic loading cases [44, 45, 46], even less in high-rate dynamics (in which wave propagation and reflection effectively leads to alternated stresses). In order to propose an anisotropic damage model suitable for such dynamics applications, the concept of active damage is defined and used in both the criterion function and the novel delay-active damage law (see sections 2.2 and 3). It is a key concept to make uncouple the rate dependent responses in tension and in compression in dynamic and impact cases. A modular damage model is then be proposed: with delay-damage or strain rate independent, with or without active damage, local or non local.

Last, regularization issues are studied (section 6), mainly from a computational point of view, for different versions of the modular anisotropic delay-damage model. Damage softening constitutive equations lead to the well-known problem of mesh dependency due to strain localization. A now classical method to deal with this difficulty is the non-local enhancement $[47,48]$. It has proven its efficiency in quasi-statics. The case of dynamics implying waves propagation still needs attention $[51,52,53,54]$. Another possibility is to introduce a time dependency in the degradation mechanism. Initially, this was a numerical tool to avoid mesh dependency, in case of plasticity [55] but also in case of continuous damage $[30,38,56,57,58]$. Within continuous damage constitutive equations, to introduce a saturating damage rate has proved quite efficient regarding regularization [39]. The modular delay-damage laws with or without active damage, local or nonlocal - proposed in present work extends such a feature to the case of induced damage anisotropy. One attempts therefore to answer to the question of its material representativity (in terms of strain rate effect reproduced) but also of its efficiency as a localization limiter (with the same identified material parameters).

\section{Initial anisotropic damage model}

\subsection{Initial model for monotonic applications}

An anisotropic damage model has been proposed in the thermodynamics framework [27, 28] introducing for quasi-brittle materials a single damage variable (the second order tensor $\boldsymbol{D}$ ) as the representation of the damage state due to micro-cracking. Mainly due to induced anisotropy, the dissymmetric response of concrete in tension and in compression is obtained with a low number of material parameters (5 including elasticity parameters). Mazars damage criterion [15] is used in this initial model, with the advantage of simplicity for instance from the numerical point of view.

The full set of constitutive equations reads

- Elasticity,

$$
\boldsymbol{\varepsilon}=\frac{1+v}{E} \tilde{\boldsymbol{\sigma}}-\frac{v}{E} \operatorname{tr} \tilde{\boldsymbol{\sigma}} \mathbf{1} \quad \text { or } \quad \boldsymbol{\varepsilon}=\mathbb{E}^{-1}: \tilde{\boldsymbol{\sigma}}
$$

with $E$ the Young modulus and $v$ the Poisson ratio.

- Effective stress ${ }^{1}$,

$$
\tilde{\boldsymbol{\sigma}}=\left[(\mathbf{1}-\boldsymbol{D})^{-1 / 2} \boldsymbol{\sigma}^{D}(\mathbf{1}-\boldsymbol{D})^{-1 / 2}\right]^{D}+\frac{1}{3}\left[\frac{\langle\operatorname{tr} \boldsymbol{\sigma}\rangle_{+}}{1-\operatorname{tr} \boldsymbol{D}}+\langle\operatorname{tr} \boldsymbol{\sigma}\rangle_{-}\right] \mathbf{1}
$$

\footnotetext{
${ }^{1}$ The power $\alpha$ of a positive symmetric second order tensor $\boldsymbol{T}$ is taken in terms of principal values, making $\boldsymbol{T}$ diagonal as $\boldsymbol{T}_{\text {diag }}=\boldsymbol{P}^{-1} \boldsymbol{T} \boldsymbol{P}$ with $\boldsymbol{P}$ the change of base matrix and finally $\boldsymbol{T}^{\alpha}=\boldsymbol{P} \boldsymbol{T}_{\text {diag }}^{\alpha} \boldsymbol{P}^{-1}$.
} 
where (.) $)^{D}$ stands for the deviatoric part of a tensor and $<.>_{+}$(resp. $<_{.}>_{-}$) for the positive (resp. negative) part of either a scalar or a tensor (in terms of principal values). Previous relationship is inverted as

$$
\boldsymbol{\sigma}=(\mathbf{1}-\boldsymbol{D})^{1 / 2} \tilde{\boldsymbol{\sigma}}(\mathbf{1}-\boldsymbol{D})^{1 / 2}-\frac{(\mathbf{1}-\boldsymbol{D}): \tilde{\boldsymbol{\sigma}}}{3-\operatorname{tr} \boldsymbol{D}}(\mathbf{1}-\boldsymbol{D})+\frac{1}{3}\left[(1-\operatorname{tr} \boldsymbol{D})\langle\operatorname{tr} \tilde{\boldsymbol{\sigma}}\rangle_{+}+\langle\operatorname{tr} \tilde{\boldsymbol{\sigma}}\rangle_{-}\right] 1
$$

- Damage criterion,

$$
f=g(\hat{\varepsilon})-\operatorname{tr} \boldsymbol{D} \quad \text { so that }\left\{\begin{array}{l}
f<0 \rightarrow \text { elastic loading or unloading } \\
f=0 \& \dot{f}=0 \rightarrow \text { damage growth }
\end{array}\right.
$$

where $\hat{\varepsilon}=\sqrt{\langle\boldsymbol{\varepsilon}\rangle_{+}:\langle\boldsymbol{\varepsilon}\rangle_{+}}$is Mazars equivalent strain and where $g(\hat{\varepsilon})=a A\left[\arctan \left(\frac{\hat{\varepsilon}}{a}\right)-\arctan \left(\frac{\kappa_{0}}{a}\right)\right]$ is used, introducing $\kappa_{0}$ as damage threshold, $A$ and $a$ as damage parameters. Note that other definitions of the equivalent strain can be used [50, 59].

- Induced damage anisotropy governed by the positive extensions ${ }^{2}$,

$$
\dot{\boldsymbol{D}}=\dot{\lambda}\langle\boldsymbol{\varepsilon}\rangle_{+}^{2}
$$

In the rate independent formulation the damage multiplier $\dot{\lambda}$ is determined from the consistency condition $f=$ $0 \& \dot{f}=0$.

\subsection{Damage deactivation for micro-cracks closure}

Splitting into positive and negative parts the hydrostatic stress in the state potential allows to reproduce naturally a partial micro-deffects closure for alternated loading (the example of tension followed by compression is given next). Gibbs state potential from which derives elasticity law (1)-(2) reads:

$$
\rho \psi^{\star}=\frac{1+v}{2 E} \operatorname{tr}\left[(\mathbf{1}-\boldsymbol{D})^{-1 / 2} \boldsymbol{\sigma}^{D}(\mathbf{1}-\boldsymbol{D})^{-1 / 2} \boldsymbol{\sigma}^{D}\right]+\frac{(1-2 v)}{6 E}\left(\frac{\langle\operatorname{tr} \boldsymbol{\sigma}\rangle_{+}^{2}}{1-\operatorname{tr} \boldsymbol{D}}+\langle\operatorname{tr} \boldsymbol{\sigma}\rangle_{-}^{2}\right)
$$

It is split into two parts, deviatoric and hydrostatic - itself also split into two parts. The hydrostatic part is not affected by damage for negative hydrostatic stresses, i.e. bulk modulus is constant in compression (compaction is not modeled). Damage acts fully on shear (deviatoric) stresses, independently of their sign. Such a potential represents then partial stiffness recovery under alternated loadings.

For a complete recovery, it would be necessary to split also the deviatoric term into a negative and a positive part [11]. This makes the numerical scheme much heavier for a limited interest. Note that the few experimental results existing on concrete behavior under alternated loading [1] are not sufficient to conclude on how complete is the stiffness recovery.

The initial model of section 2.1 is not able to deal properly with alternated loadings since the damage threshold in terms of strains $\kappa(\operatorname{tr} \boldsymbol{D})=g^{-1}(\operatorname{tr} \boldsymbol{D})$ acts as the isotropic hardening of metals plasticity, but in the strains space. Isotropic hardening is known as not sufficient to model cyclic plasticity (kinematic hardening is usually introduced for such a purpose). With $\kappa(\operatorname{tr} \boldsymbol{D})$ consolidation function, the threshold is reached in compression (resp. in tension) for a larger strain (in absolute value) after a damaging loading in tension (resp. in compression) than for a monotonic loading (Fig. 1(a)). In order to solve this problem - similar to the one encountered when modeling metals Bauschinger effect - the concept of active damage is introduced, denoted $d_{a c t}$ and defined as follows:

$$
d_{a c t}=\frac{\boldsymbol{D}:\langle\boldsymbol{\varepsilon}\rangle_{+}}{\max _{I} \varepsilon_{I}}=\frac{D_{i j}\left(\langle\boldsymbol{\varepsilon}\rangle_{+}\right)_{i j}}{\max _{I} \varepsilon_{I}}
$$

\footnotetext{
${ }^{2}$ The positive part of a second order tensor $\boldsymbol{T}$ is taken in terms of principal values, making $\boldsymbol{T}$ diagonal, positive diagonal as $\left\langle\boldsymbol{T}_{\text {diag }}\right\rangle_{+}=\left\langle\boldsymbol{P}^{-1} \boldsymbol{T P}\right\rangle_{+}$ and finally $\langle\boldsymbol{T}\rangle_{+}=\boldsymbol{P}\left\langle\boldsymbol{T}_{\text {diag }}\right\rangle_{+} \boldsymbol{P}^{-1}$.
} 
with $\max _{I} \varepsilon_{I}$ the maximum principal extension. Definition (7) is such as $d_{a c t}=\operatorname{tr} \boldsymbol{D}$ in monotonic tension as well as in monotonic compression. The damage threshold is then rewritten:

$$
f=g(\hat{\varepsilon})-d_{a c t}=0
$$

keeping then unchanged the material parameters of initial model. The damage threshold is now reached for the same equivalent strain in a compression (resp. a tension) following a damaging tension loading (resp. compression) than in the monotonic compression case (resp. tension). Figure 1(b) shows the damage deactivation effect on an alternated loading tension - up to a damage amount in loading direction $D_{1}$ - followed by compression. The peak stress in compression is well reduced when the compression follows a damaging tension. A contrario, note that the ultimate strain in compression (at vanishing stress) is not affected by the amount of damage $D_{1}$ reached in tension.

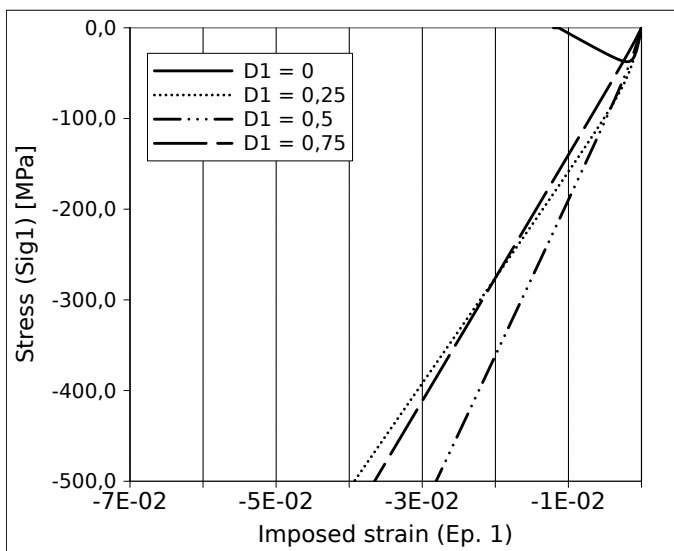

(a) Without damage deactivation

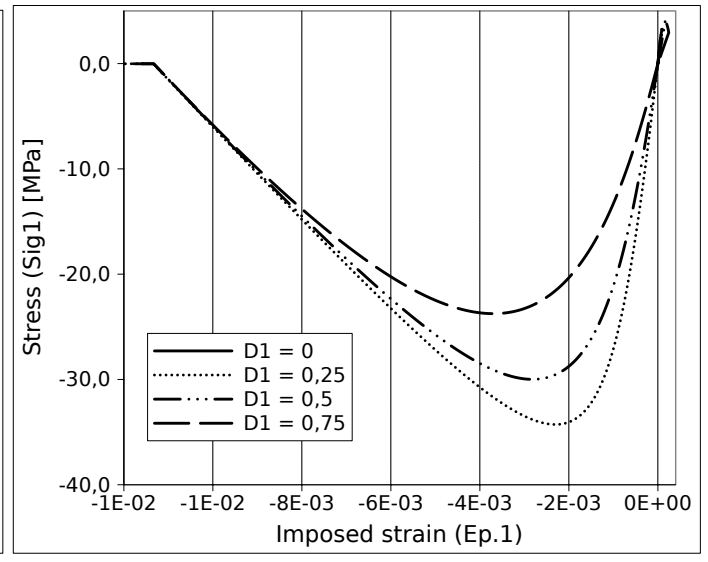

(b) With damage deactivation

Figure 1: Effect of damage deactivation on a tension up to $D_{1}$ followed by compression loading.

\subsection{Extension to nonlocal}

Classical mesh dependency occurs when using previous local damage model in a Finite Element code. A non-local enhancement can be used to gain the mesh independency. One just has to replace local Mazars strain $\hat{\varepsilon}$ in the damage criterion $f$ by nonlocal strain $\hat{\varepsilon}^{n l}$ and to set

$$
f=g\left(\hat{\varepsilon}^{n l}\right)-d_{a c t}
$$

Mazars nonlocal equivalent strain can be defined by use of an integral form (next with a Gaussian nonlocal weight function) or of an implicit second gradient form. One uses here the non-local integral averaging over the whole domain [47]:

$$
\begin{aligned}
& \hat{\varepsilon}^{n l}=\hat{\varepsilon}^{n l}(\mathbf{x})=\frac{1}{V_{r}} \int_{\Omega} \exp \left(-\frac{4\|\mathbf{x}-\mathbf{s}\|^{2}}{l_{c}^{2}}\right) \hat{\varepsilon}(\mathbf{s}) d s \\
& V_{r}(\mathbf{x})=\int_{\Omega} \exp \left(-\frac{4\|\mathbf{x}-\mathbf{s}\|^{2}}{l_{c}^{2}}\right) d s
\end{aligned}
$$

with $l_{c}$ the characteristic length. Let us mention the implicit gradient enhancement $[60,48]$

$$
\hat{\varepsilon}^{n l}-l_{c}^{2} \nabla^{2} \hat{\varepsilon}^{n l}=\hat{\varepsilon}
$$

which can be used instead of Eq. (10). 


\section{Anisotropic delay-damage model}

Experiments show that concrete tensile strength can be multiplied by a factor of 10 or more for high strain rates (high meaning of the order of $\dot{\varepsilon}=150 \mathrm{~s}^{-1}$ [35]) while in compression the dynamic increase factor - the ratio $f_{c}^{d y n} / f_{c}$ of the dynamic strength to the quasi-static one - does not exceed 2 or 3 . In compression, strain rate effects can be explained at high rates by inertia consideration $[61,62,63]$ : internal forces due to acceleration lead to a confined stress state which makes concrete more ductile and resistant [64]. In tension at low strain rates, the strength enhancement can be related to viscous effects due the presence of water inside the cement matrix [65], while when the strain rate is increased there is a transition from a single macro-crack failure to a multiple cracking pattern due to defects obscuration mechanisms [69]. It is chosen next to reproduce the strain rate effect in compression simply by considering the inertial terms in the global equilibrium. The only "material" strain rate effect which will need modeling will then be the tensile one. Different possibilities exist in the litterature, depending on the deformation mechanism considered, visco-plasticity [66, 41], visco-elasticity [42], viscous hardening [40], visco-damage [9, 38]. In present work, the time dependency is introduced through a viscosity law for damage evolution, extended to induced anisotropy.

\subsection{Visco/delay-damage laws}

A modeling possibility for high-rate dynamics and impact consists in making the strain rate affect the stress-strain response of concrete. The introduction of a characteristic time in dynamics indirectly defines a characteristic length. In the present case of elasticity coupled with damage this is done by introducing a viscosity or visco-damage law $D_{v}=D_{v}(\operatorname{tr} \dot{\boldsymbol{D}})$ in Mazars criterion [67, 68]. The damage evolution occurs not anymore at $f=0$ but at $f=D_{v}>0$. A classical law for isotropic damage is Norton-Perzyna power law, $D_{v}=k \dot{D}^{1 / m}$, with $k$ and $m$ the visco-damage parameters [38]. Such a law leads to an unbounded damage rate often too important at high strain rates.

It is possible to bound the damage rate, for instance by the maximum rate $\dot{D}_{\infty}=1 / \tau_{b}$, a material parameter equal to the inverse of a characteristic time $\tau_{b}$ [39]. To gain this property in the thermodynamics framework one sets

$$
f=g(\hat{\varepsilon})-\operatorname{tr} \boldsymbol{D}=D_{v}>0 \quad D_{v}=-\frac{1}{b} \ln \left(\frac{\dot{D}_{\infty}-\operatorname{tr} \dot{\boldsymbol{D}}}{\dot{D}_{\infty}}\right)
$$

from which derives the delay-damage law extended in induced damage anisotropy

$$
\operatorname{tr} \dot{\boldsymbol{D}}=\dot{D}_{\infty}[1-\exp (-b(g(\hat{\varepsilon})-\operatorname{tr} \boldsymbol{D}))]
$$

bounding tr $\dot{\boldsymbol{D}}$ at high strain rates.

This regularization is defined locally (i.e. at RVE scale, at a structure Gauss point in Finite Elements) and is well adapted for dynamics computations. It allows for the determination of the damage multiplier $\dot{\lambda}$ (of eq. 4 ) and replaces the consistency condition $f=0, \dot{f}=0$ of the rate independent case.

Note that for small values of the criterion function $f=g(\hat{\varepsilon})-\operatorname{tr} \boldsymbol{D}$ the exponential simplifies as the linear law $(m=1)$ :

$$
1-\exp (-b f) \approx b f \quad \rightarrow \quad f=D_{v} \approx \frac{1}{b \dot{D}_{\infty}} \operatorname{tr} \dot{\boldsymbol{D}}
$$

Expression (13) models the strain rate effect encountered in concrete materials as shown in figure 2. The viscous effect obtained with law (13) is of the same order of magnitude in tension and in compression when material evidences exhibit a smaller effect in compression. For quasi-brittle materials, one proposes then the following exponential law,

$$
\mathcal{H}(\operatorname{tr} \boldsymbol{\varepsilon}) \operatorname{tr} \dot{\boldsymbol{D}}=\dot{D}_{\infty}[1-\exp (-b(g(\hat{\varepsilon})-\operatorname{tr} \boldsymbol{D}))]
$$

which bounds the damage rate for positive hydrostatic strains and which deactivates the delay effect for negative hydrostatic strains $(\mathcal{H}(x)=1$ if $x \geqslant 0, \mathcal{H}(x)=0$ otherwise is Heaviside function). 


\subsection{Damages deactivation in dynamics}

In dynamics, loading sign changes are frequently encountered due to wave propagation. Following the developments of section 2.2 for quasi-static loadings, the active damage can be introduced in the delay-damage model, both in the damage threshold and the viscosity law, in order to deal with alternated loadings. It is possible in these cases to make the damage state due to compression (previously represented by $\operatorname{tr} \boldsymbol{D}$ ) not affect the strain rate effect in tension. This is a key point to properly model with anisotropic damage waves induced dynamics failure. In that purpose, the novel anisotropic delay-damage evolution law is written in terms of active damage $d_{a c t}$ (defined by Eq. (7)):

$$
\mathcal{H}(\operatorname{tr} \boldsymbol{\varepsilon}) \dot{d}_{a c t}=\dot{D}_{\infty}\left[1-\exp \left(-b\left(g(\hat{\varepsilon})-d_{a c t}\right)\right)\right]
$$

\subsection{Constitutive equations of delay-active damage model}

The full set of constitutive equations now reads

- Elasticity

$$
\boldsymbol{\varepsilon}=\frac{1+v}{E}\left[(\mathbf{1}-\boldsymbol{D})^{-1 / 2} \boldsymbol{\sigma}^{D}(\mathbf{1}-\boldsymbol{D})^{-1 / 2}\right]^{D}+\frac{1-2 v}{3 E}\left[\frac{\langle\operatorname{tr} \boldsymbol{\sigma}\rangle_{+}}{1-\operatorname{tr} \boldsymbol{D}}+\langle\operatorname{tr} \boldsymbol{\sigma}\rangle_{-}\right] \mathbf{1}
$$

- Damage criterion (local) $f=g(\hat{\varepsilon})-d_{\text {act }}$, using the viscous regularization (12),

$$
\begin{aligned}
& f \leq 0 \rightarrow \text { elastic loading or unloading } \\
& f=D_{v}>0 \rightarrow \text { damage growth }
\end{aligned}
$$

- Induced damage anisotropy governed by the positive extensions, damage multiplier $\dot{\lambda}$ being determined from $f=D_{v}\left(d_{a c t}\right)$ rewritten as Eq. (16)

$$
\dot{\boldsymbol{D}}=\dot{\lambda}\langle\boldsymbol{\varepsilon}\rangle_{+}
$$

Note that a non-local model, is gained simply by replacing $\hat{\varepsilon}$ by non-local equivalent strain $\hat{\varepsilon}^{\text {nl }}$ (Eq. 10) in damage criterion:

$$
f=g\left(\hat{\varepsilon}^{n l}\right)-d_{a c t}
$$

The non-local delay-active damage evolution law is then:

$$
\mathcal{H}(\operatorname{tr}(\boldsymbol{\varepsilon})) \dot{d}_{a c t}=\dot{D}_{\infty}\left[1-\exp \left(-b\left(g\left(\hat{\varepsilon}^{n l}\right)-d_{a c t}\right)\right)\right]
$$

\subsection{Positivity of the intrinsic dissipation}

It has been shown in previous works $[70,28]$ that the local initial model of section 2.1 was thermodynamically consistent, for instance that it leads to an intrinsic dissipation due to damage $\mathcal{D}=\boldsymbol{Y}: \dot{\boldsymbol{D}} \geq 0$ positive for any loading, even non proportional, even random. The proof, not recalled here, is valid for a family of thermodynamics potential $\rho \psi^{\star}$ - including potential (6) - and does not need the explicit derivation of the thermodynamics force $\boldsymbol{Y}=\rho \frac{\partial \psi^{\star}}{\partial \boldsymbol{D}}$. It just needs the feature $\dot{\boldsymbol{D}} \geq 0$ of a positive (tensorial) damage rate and applies to non standard damage models, for which the damage evolution law is not gained by normality from a convex criterion function $f$.

For the delay-damage model, the damage rate $\dot{\boldsymbol{D}}=\dot{\lambda}\langle\boldsymbol{\varepsilon}\rangle_{+}$is a positive tensor, built from the positive eigenstrains, as the damage multiplier $\dot{\lambda}$ is as usual positive (when $f=D_{v} \geq 0$, damage growth) or zero (when $f<0$, elastic unloading).

The case of non local modeling is more complex in the general case as it strongly depends on the thermodynamics formulation for the nonlocal enhancement [71, 48, 50]. A comparison of nonlocal and gradient-enhanced softening continua can be found in [49] (it is shown for instance that wave velocity is unbounded for the explicit gradient enhancement, which is not a physical feature). Strain localization and dispersive wave propagation in softening plastic Cosserat media has been studied in [72]. A recent review on the implicit gradient thermodynamics formulation [73] summarizes the different approaches (called micromorphic in Forest's work). A rigorous formulation recovering Eq. (11) considers the variable $\hat{e}$ and gradient variable $\nabla \hat{e}$ as additional state (thermodynamics) variables. Following 
[74] and [73], $\hat{e}$ is derived from virtual work consideration as equal to $\hat{\varepsilon}^{n l}$ given by Eq. (11). In order to allow this, note that Hemholtz free energy becomes (with $\alpha$ and $l_{c}$ as material parameters),

$$
\rho \psi^{n l}=\rho \psi^{l o c a l}(\boldsymbol{\varepsilon}, \boldsymbol{D})+\frac{\alpha}{2}\left[(\hat{\varepsilon}-\hat{e})^{2}+l_{c}^{2} \nabla \hat{e} \cdot \nabla \hat{e}\right]
$$

with $\rho \psi^{\text {local }}=\sup _{\boldsymbol{\sigma}}\left(\boldsymbol{\sigma}: \boldsymbol{\varepsilon}-\rho \psi^{\star}\right)$ gained by Legendre transform of Gibbs free enthalpy (6). Note that the elasticity law is changed into a nonlocal one as

$$
\boldsymbol{\sigma}=\rho \frac{\partial \psi^{\text {local }}}{\partial \boldsymbol{\varepsilon}}+\alpha(\hat{\varepsilon}-\hat{e}) \frac{\partial \hat{\varepsilon}}{\partial \boldsymbol{\varepsilon}}=\rho \frac{\partial \psi^{\text {local }}}{\partial \boldsymbol{\varepsilon}}+\alpha \frac{\hat{\varepsilon}-\hat{\varepsilon}^{n l}}{\hat{\varepsilon}}\langle\boldsymbol{\varepsilon}\rangle_{+}
$$

which recovers Eq. (17) only if a small value for $\alpha$ is considered. Another pragmatic formulation simply postulates the non-local enhancement (for example as Eq. (11) or as the integral averaging (10)) and does not derive it from virtual work consideration.

In both cases, the (conjugate) thermodynamics force $\boldsymbol{Y}=-\rho \frac{\partial \psi^{n l}}{\partial \boldsymbol{D}}=-\rho \frac{\partial \psi^{l o c a l}}{\partial \boldsymbol{D}}=\rho \frac{\partial \psi^{\star}}{\partial \boldsymbol{D}}$ remains unchanged compared to the local model. And it is still defined in a local manner. The proof [70] of the positivity of the dissipation due to damage holds then. Recall that for thermodynamics potential (6) it only needs $\dot{\boldsymbol{D}} \geq 0$ (as one only needs a positive scalar rate $\dot{D} \geq 0$ in case of nonlocal isotropic damage [74]). The second principle of thermodynamics is fulfilled for the nonlocal anisotropic delay-damage model.

\section{Strain rate effect and its consequence on damage growth}

\subsection{Monotonic loading}

Experiments made either on hydraulic testing machines at different controled strain rates, or performed with Hopkinson bars at larger strain rates, exhibit the so-called rate effect: the larger the strain rate the larger the apparent tensile $f_{t}^{d y n}$ or compressive $f_{c}^{d y n}$ strengths. The results for concrete are gathered in the strength increase $f_{t}^{d y n} / f_{t}$ and $f_{c}^{d y n} / f_{c}$ vs $\dot{\varepsilon}$ curves of figures 2 and 3 , with $f_{t}=f_{t}(\dot{\epsilon} \approx 0), f_{c}=f_{c}(\dot{\epsilon} \approx 0)$ the quasi-statics ultimate stresses in tension and in compression.

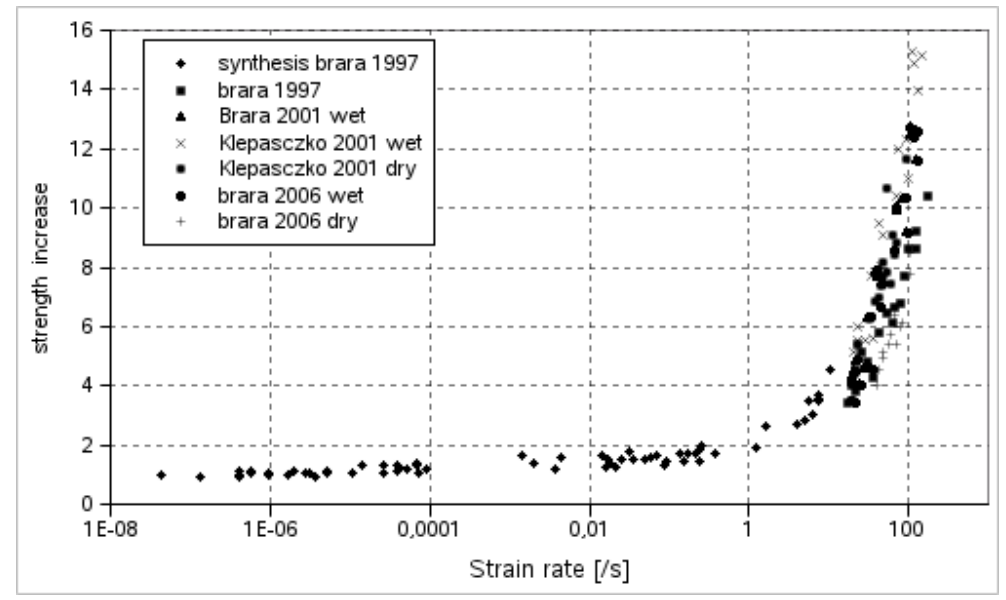

Figure 2: Strain rate effect in tension for concrete , after [32, 33, 34, 35, 36].

As already mentioned, the strength increase observed in compression is weaker than in tension (Fig. 3) and can be related to inertia effects that confine the material. This same enhancement is simply reproduced by Finite Elements if inertial forces are considered (Fig. 4). The model of section 3 has been used for this computation with the set of material parameters: $E=42 \mathrm{GPa}, v=0.2, \kappa_{0}=5.10^{-5}, A=5000, a=2.93 .10^{-3}, \dot{D}_{\infty}=50000 \mathrm{~s}^{-1}$ and $b=1$. 


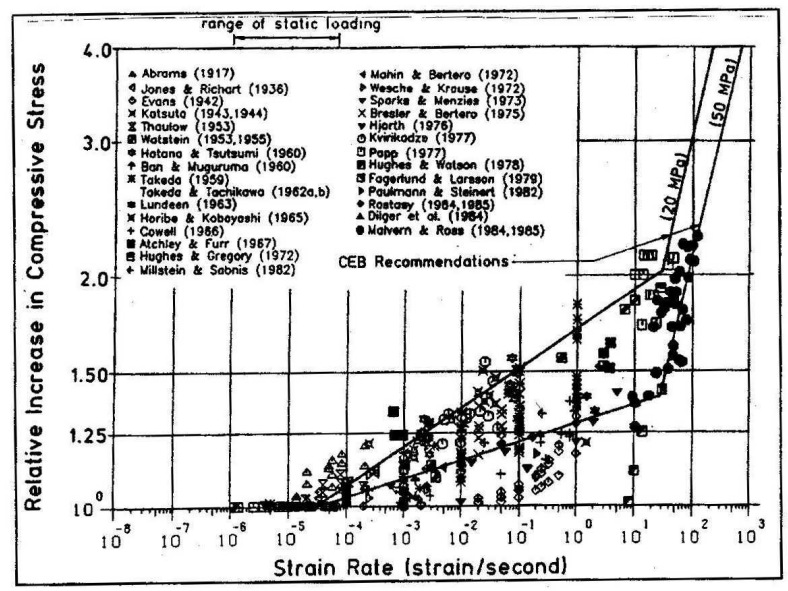

Figure 3: strain rate effect in compression for concrete, after [31].

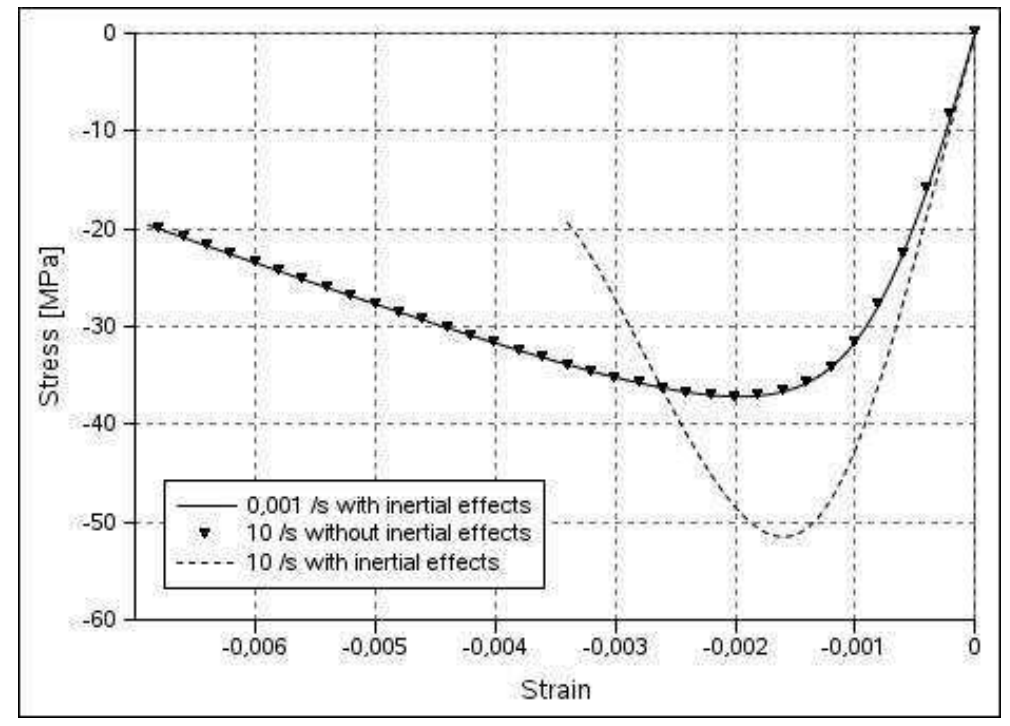

Figure 4: Inertia effect in compression .

The computation is performed on a single cubic element loaded in uniaxial compression. Recall that damage evolution occurs without viscosity in compression (neither from delay-damage law (15) nor from law (16) due to $\mathcal{H}(\operatorname{tr} \boldsymbol{\varepsilon})=0)$. In tension $(\mathcal{H}(\operatorname{tr} \boldsymbol{\varepsilon})=1)$, delay-damage allows to represent the strain rate effect shown in Fig. 2 . It is exactly the same without or with active damage (as long as there is no initial compressive stage).

The two delay-damage parameters, $\dot{D}_{\infty}$ and $b$, have been identified such as one has simultaneously a noticeable strength increase starting from a strain rate of about $1 \mathrm{~s}^{-1}$ and still a brittle softening behavior at $200 \mathrm{~s}^{-1}$ (cf. Fig. 5). A good fit for the strain rate effect is obtained with the common value $b=1$ and with $\dot{D}_{\infty}=50000 \mathrm{~s}^{-1}$ (which corresponds to a characteristic time $\tau_{b}=2 \cdot 5 \cdot 10^{-5} \mathrm{~s}$ ).

Further investigations on the consequences of such a modeling on computations of impacted structures are presented in section 6. 


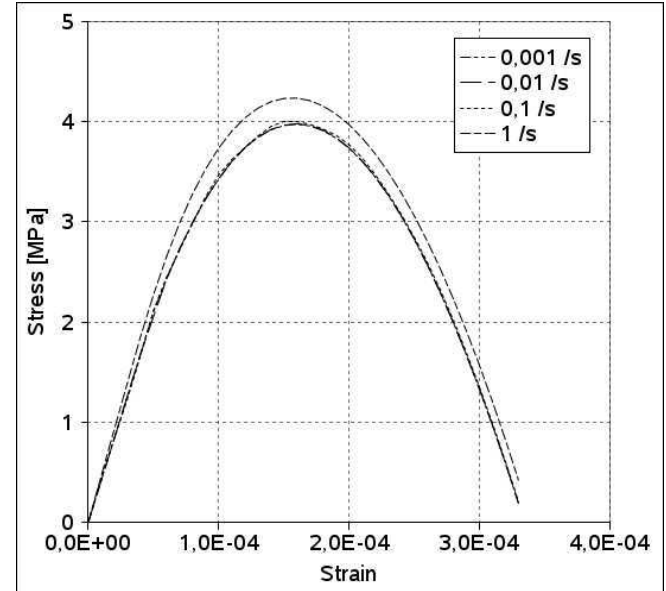

(a) low strain rates

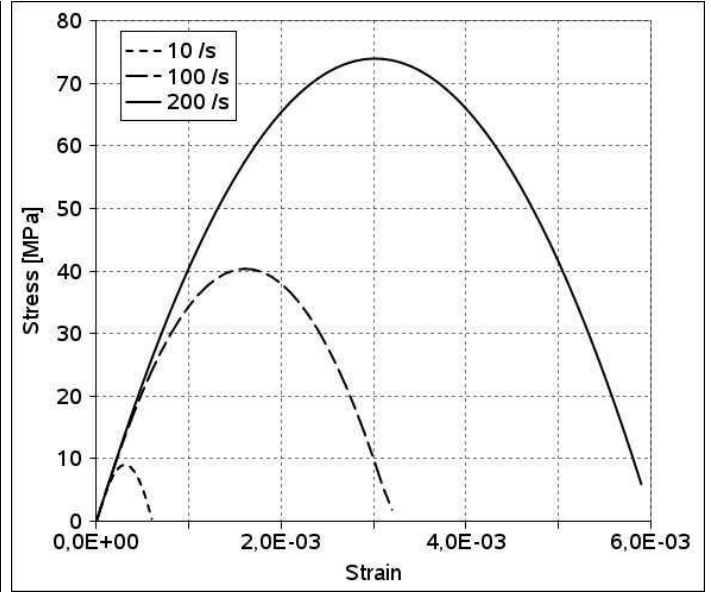

(b) high strain rates

Figure 5: Calculated stress-strain curves at different strain rates (anisotropic delay-damage model).

\subsection{Alternated loading}

Figure 6 shows that the deactivation of damage in dynamics (i.e. the use of $d_{a c t}$ in the delay-damage law, Eq. 21) allows to reproduce a physically consistent strain rate effect in tension after a first damaging stage in compression. The example presented consists in a numerical simulation carried out on a single cubic element first damaged in compression along direction 1 until

$$
\boldsymbol{D}=\left(\begin{array}{ccc}
0 & 0 & 0 \\
0 & 0.5 & 0 \\
0 & 0 & 0.5
\end{array}\right)
$$

and then loaded in tension at a strain rate of $\dot{\varepsilon}_{11}=10 \mathrm{~s}^{-1}$. The numerical response for a monotonic tension loading at the same strain rate is also represented for comparison. When the law in $\operatorname{tr} \boldsymbol{D}$ is used (Eq. 15, without active damage), the strength reached in dynamic tension is found larger $(\simeq 25 \mathrm{MPa})$ after a damaging compression than in case of the undamaged material $(\simeq 10 \mathrm{MPa})$. This is quite not realistic ! With damage deactivation (law 16), the strength enhancement in dynamics is found of the same order of magnitude with or without the initial damaging compressive stage. This shows the importance of the active damage concept (7) for dynamics.

\section{Numerical implementation in a Finite Flement code}

The numerical implementation in a Finite Element computer code can take advantage of the strain form of the damage criterion in order to avoid the use of an iterative process at the local Gauss point level. This key feature is of most importance in explicit dynamics as many (small) time steps are needed. The scheme proposed next for the anisotropic delay-active damage is detailed in two steps: one first focus on the delay-damage law time discretization, second on the numerical scheme for the full anisotropic damage model. The strain $\boldsymbol{\varepsilon}_{n+1}$ at time $t_{n+1}$ is assumed known as well as quantities at time $t_{n}$, and one seeks the damage $\boldsymbol{D}_{n+1}$ and stress $\boldsymbol{\sigma}_{n+1}$.

\subsection{An explicited Euler backward scheme for delay-damage law}

Let us consider a damaging increment.

The case of negative hydrostatic ( $\operatorname{tr} \varepsilon \leq 0$ ) leads to the same resolution as in quasi-statics as one has then $\mathcal{H}(\operatorname{tr} \boldsymbol{\varepsilon})$ $=0$ and:

$$
d_{a c t n+1}=g\left(\hat{\varepsilon}_{n+1}\right)
$$




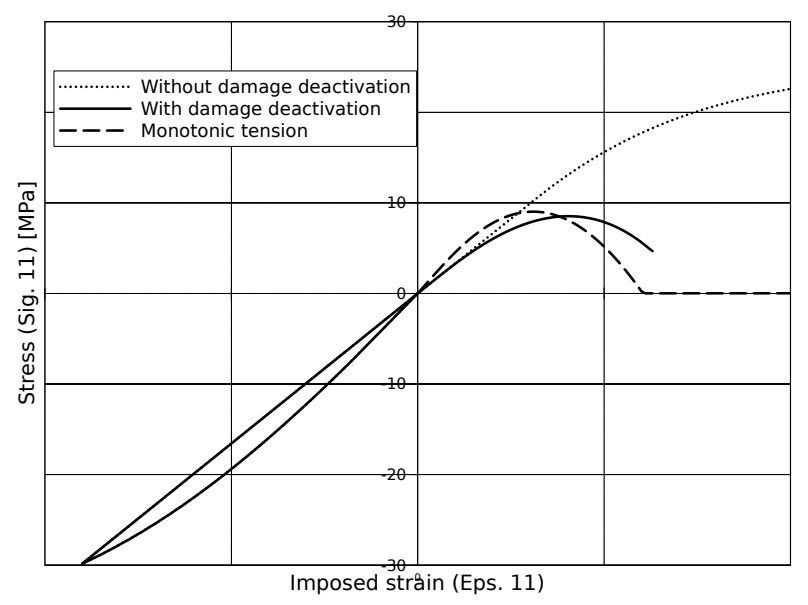

Figure 6: Effect of damage deactivation on the tensile strain rate effect $\left(\dot{\epsilon}=10 \mathrm{~s}^{-1}\right)$.

In case of positive hydrostatic strain ( $\operatorname{tr} \varepsilon \geqslant 0$ ), the derivative with respect to time of the delay-damage law (16) leads to:

$$
g^{\prime}(\hat{\varepsilon}) \dot{\hat{\varepsilon}}-\dot{d}_{a c t}=\frac{1}{b} \frac{\ddot{d}_{a c t}}{\dot{D}_{\infty}-\dot{d}_{a c t}}
$$

with here

$$
g^{\prime}(\hat{\varepsilon})=\frac{A}{1+\left(\frac{\hat{\varepsilon}}{a}\right)^{2}}
$$

Using Euler backward scheme,

$$
\begin{aligned}
\ddot{d}_{a c t n+1} & \approx \frac{\dot{d}_{a c t n+1}-\dot{d}_{a c t n}}{\Delta t} \\
\dot{\hat{\varepsilon}}_{n+1} & \approx \frac{\hat{\varepsilon}_{n+1}-\hat{\varepsilon}_{n}}{\Delta t}
\end{aligned}
$$

equation (25) becomes

$$
\left(\dot{d}_{a c t n+1}\right)^{2}-\left(g^{\prime}\left(\hat{\varepsilon}_{n+1}\right) \dot{\hat{\varepsilon}}_{n+1}+\dot{D}_{\infty}+\frac{1}{b \Delta t}\right) \dot{d}_{a c t n+1}+g^{\prime}\left(\hat{\varepsilon}_{n+1}\right) \dot{\hat{\varepsilon}}_{n+1} \dot{D}_{\infty}+\frac{\dot{d}_{a c t n}}{b \Delta t}=0
$$

so that the active damage rate at time $t_{n+1}$ is solution of the second degree equation (here in a form which avoids the difference of large numbers),

$$
\begin{gathered}
\dot{d}_{a c t n+1}=\frac{1}{2} B_{n+1}\left[1-\sqrt{1-4 \frac{C_{n+1}}{B_{n+1}^{2}}\left(1+\frac{\dot{d}_{a c t n}}{b \Delta t C_{n+1}}\right)}\right] \\
d_{a c t n+1}=d_{a c t n}+\Delta t \dot{d}_{a c t n+1}
\end{gathered}
$$

with as initial conditions $d_{a c t 0}=0, \dot{d}_{a c t 0}=0$ and where:

$$
\begin{aligned}
& B_{n+1}=g^{\prime}\left(\hat{\varepsilon}_{n+1}\right) \dot{\hat{\varepsilon}}_{n+1}+\dot{D}_{\infty}+\frac{1}{b \Delta t} \\
& C_{n+1}=g^{\prime}\left(\hat{\varepsilon}_{n+1}\right) \dot{\hat{\varepsilon}}_{n+1} \dot{D}_{\infty}
\end{aligned}
$$




\subsection{Explicited Euler backward scheme for anisotropic delay-active damage model}

The time integration procedure for the full anisotropic delay-damage model takes place at a Gauss point and solves in a coupled manner the constitutive equations of section 2. The strain $\boldsymbol{\varepsilon}_{n+1}=\boldsymbol{\varepsilon}\left(t_{n+1}\right)$ at time $t_{n+1}$, the damage $\boldsymbol{D}_{n}$ and the active damage rate $\dot{d}_{a c t n}$ at time $t_{n}$ are the inputs of the procedure. The outputs are the stresses $\boldsymbol{\sigma}_{n+1}$ and the damage $\boldsymbol{D}_{n+1}$, but also the active damage rate $\dot{d}_{a c t n+1}$ at time $t_{n+1}$. Euler backward implicit scheme is used for stability reason, i.e. the variables are replaced by their value at time $t_{n+1}$ in the constitutive equations when the damage rate $\dot{\boldsymbol{D}}$ and the damage multiplier $\dot{\lambda}$ are replaced by $\Delta \boldsymbol{D}=\boldsymbol{D}_{n+1}-\boldsymbol{D}_{n}$ and $\Delta \lambda=\lambda_{n+1}-\lambda_{n}$ in the damage law. In order to integrate the damage model proceed as follows:

1. Compute the equivalent strain $\hat{\varepsilon}_{n+1}=\sqrt{\left.\left\langle\boldsymbol{\varepsilon}_{n+1}\right\rangle_{+}:<\boldsymbol{\varepsilon}_{n+1}\right\rangle_{+}}$

2. Make a test on the criterion function $f=g\left(\hat{\varepsilon}_{n+1}\right)-d_{a c t n}$

- If $f \leq 0$, the material behaves elastically, set then $\boldsymbol{D}_{n+1}=\boldsymbol{D}_{n}, \dot{d}_{a c t n}=0$ and $d_{a c t n+1}=d_{a c t n}$

- If $f>0$, the damage must be corrected by using the damage evolution law discretized as $\Delta \boldsymbol{D}=$ $\boldsymbol{D}_{n+1}-\boldsymbol{D}_{n}=\Delta \lambda\left\langle\boldsymbol{\varepsilon}_{n+1}\right\rangle_{+}$. Gaining $d_{a c t n+1}$ from equation (24) if $\operatorname{tr} \boldsymbol{\varepsilon}_{n+1}<0$, from equations (30)-(33) if $\operatorname{tr} \boldsymbol{\varepsilon}_{n+1} \geq 0$, allows to derive the exact expression for the damage multiplier increment $\Delta \lambda$,

$$
\Delta \lambda=\frac{\left(\max \varepsilon_{I n+1}\right) d_{a c t n+1}-\boldsymbol{D}_{n}:\left\langle\varepsilon_{n+1}\right\rangle_{+}}{\hat{\varepsilon}_{n+1}^{2}}
$$

and the actualization of the damage tensor $\boldsymbol{D}$,

$$
\boldsymbol{D}_{n+1}=\boldsymbol{D}_{n}+\Delta \lambda\left\langle\boldsymbol{\varepsilon}_{n+1}\right\rangle_{+}
$$

3. Compute the stresses using first the elasticity law written $\tilde{\boldsymbol{\sigma}}_{n+1}=\mathbb{E}: \boldsymbol{\varepsilon}_{n+1}$, with $\mathbb{E}$ isotropic Hooke's tensor. Using then eq. (3) with $\boldsymbol{\sigma}=\sigma_{n+1}, \tilde{\boldsymbol{\sigma}}=\tilde{\boldsymbol{\sigma}}_{n+1}, \boldsymbol{D}=\boldsymbol{D}_{n+1}$ finally gives the stress tensor at the end of the increment $\sigma_{n+1}$.

The complete numerical scheme is fully implicit (it is Euler backward scheme) therefore robust and stable, but it has the main advantage of the explicit schemes: there is no need for a local iterative process as the exact solution of the discretized constitutive equations are analytically explicited.

When damage reaches large values, one must be carefull to ensure the damaged elastic tensor to remain positively defined. This is done for induced anisotropic damage by using a specific procedure for the numerical control of rupture [28].

\section{Dynamic tensile test by spalling}

\subsection{Experimental set-up and 3D Finite Elements computations}

The example developed here is the numerical simulation of a dynamic tensile test. This setup has been developed in order to obtain the tensile strength of concrete at high strain rate [35]. Its principle is described in figure 7. The setup consists in a striker (launched at the velocity $V$ ), an input bar and the tested specimen. The input bar of the experimental set up has a diameter of $40 \mathrm{~mm}$ for a $100 \mathrm{~mm}$ length, while the concrete sample has the same diameter for a length of $120 \mathrm{~mm}$. After the impact of the striker at the right end, an incident stress wave propagates in the input bar. One part of the wave is transmitted into the specimen and another one is reflected at the bar/specimen interface. The transmitted compression wave is reflected at the free end and becomes a tensile stress wave. Because of the dissymmetric tension/compression material behavior, this leads to fracture of the specimen in the spall plane.

The first computations presented here reproduce the experimental conditions (specimen size and impact velocity). The applied loading consists in an imposed motion of the impacted (right) face of the specimen. The motion evolution is gained from experimental datas, the loading function is presented in figure 8 . The $3 \mathrm{D}$ mesh is composed of prismatic 


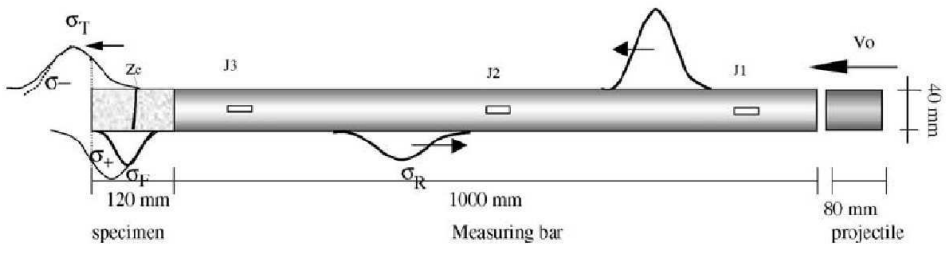

Figure 7: Principle of the dynamic tensile test, from [35]

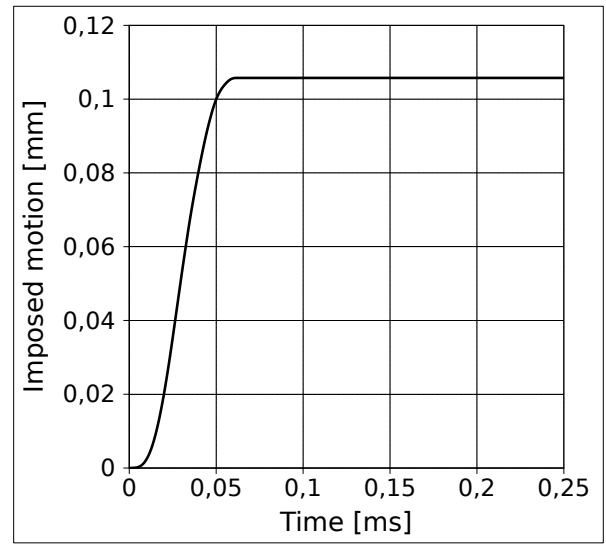

Figure 8: Numerical loading: imposed motion of the impacted face

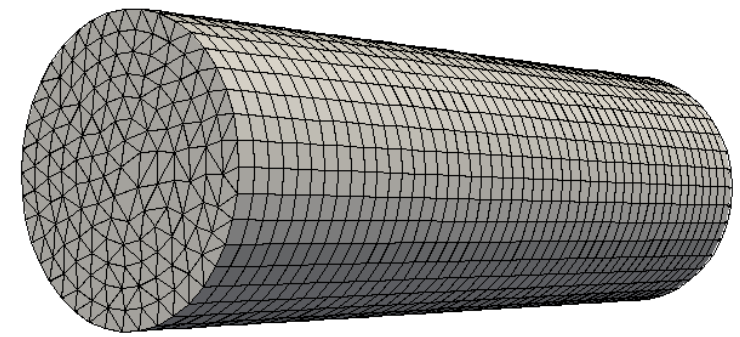

Figure 9: 3D mesh for numerical simulation of dynamic tensile test

elements with 6 nodes. There are 48 elements in the direction of the specimen length (elements length $=2.5 \mathrm{~mm}$ ). The mesh is represented in figure 9.

Experimentally, one observes that the main crack appears at $5.42 \mathrm{~cm}$ from the impacted face [36]. The experimental material parameters are not known with accuracy, the parameters taken for the model are given in table 1.

\subsection{Strain rate and micro-cracks closure effects in impacted structures}

This example illustrates the role of the strain rate and of the micro-cracks closure effect since the macro-crack position is strongly dependent on the concrete tensile strength in dynamics, which is, as mentioned before, influenced by the consideration of these two phenomenons. Indeed, the strain rate in this example is of the order of $100 \mathrm{~s}^{-1}$, a 


\begin{tabular}{|c|c|}
\hline Parameter & Value \\
\hline$E$ & $42 \mathrm{GPa}$ \\
\hline$v$ & 0.2 \\
\hline$\kappa_{0}$ & $510^{-5}$ \\
\hline$A$ & 5000 \\
\hline$a$ & $2.9310^{-4}$ \\
\hline If strain rate effect & \\
\hline$b$ & 1 \\
\hline$\dot{D}_{\infty}$ & $50000 \mathrm{~s}^{-1}$ \\
\hline
\end{tabular}

Table 1: Parameters for the anisotropic delay-damage model

value for which the strain rate effect is very influent. Furthermore and as shown in figure 10 the incident compression wave (along $x$-axis) strongly damages the specimen. The damage in compression reaches $D_{y y}=D_{z z}=0.75$ (with $D_{x x}=0$ ) in the most damaged zone. This means that the problem of a much too high apparent concrete strength in tension following compression is encountered if damage deactivation is not considered (recall that damage deactivation through the consideration of active damage $d_{a c t}$ represents in a macroscopic way the micro-cracks closure). One can notice that the specimen is not damaged near to the free face. In this zone, due to the reflexion of the incident wave, compression and tension waves are superimposed and lead to a quasi vanishing stress state at this stage.

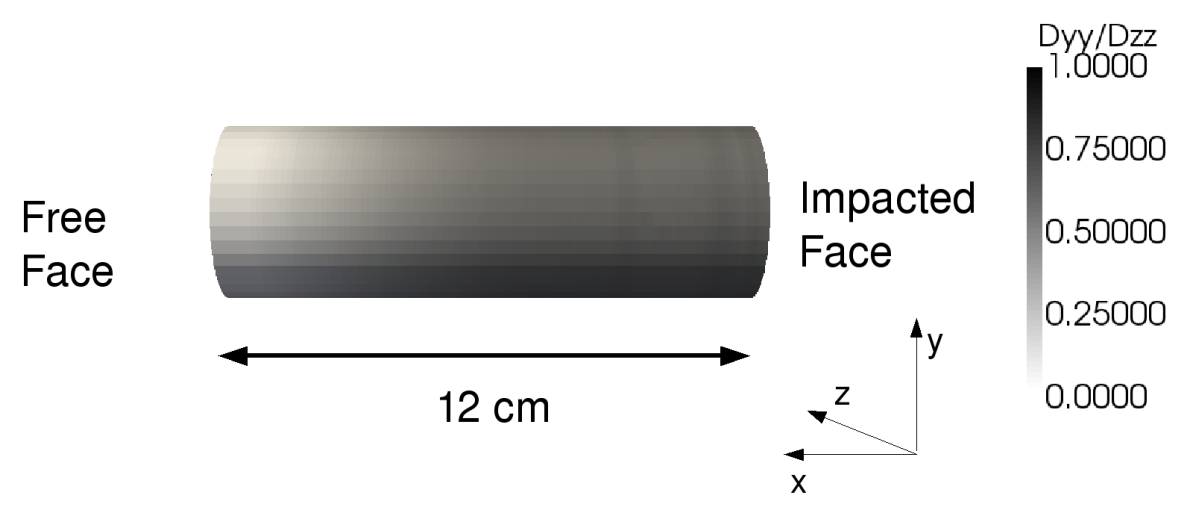

Figure 10: Damage map $D_{y y}=D_{z z}$ after the incident compression wave

Let us compare computations with four versions of the modular anisotropic damage model: with and without strain rate effect and with and without damage deactivation. The damage maps for $D_{x x}$ (due to tension) at the times corresponding to failure for the different model versions are given in figure 11. One can see that the position of the crack and the time at which failure occurs depend on the version of the model chosen, i.e. on if the strain rate effect is represented or not, if damage deactivation is considered or not. Strain rate effect leads to a later failure in an area more distant to the (left) free face because the maximal strength is tension is enlarged. If one compares the two models without damage deactivation to the experimental result, one observes that the major crack is too close to the free face in the version without delay-damage, and too far from it with delay-damage written in $\operatorname{tr} \boldsymbol{D}$. Damage deactivation allows then to correct the position of failure. With both damage deactivation and delay-damage, failure occurs close to the middle of the specimen, which fits well with the experimental result. It is important to notice that without damage deactivation, failure occurs in the less resistant area, which is, for the delay-active damage model the area not affected by damage in compression (close to the free face). In that case, the principal element that governs the position of the crack is more damage state (through the tracetr $\boldsymbol{D}$ of the damage tensor) than the strength enhancement due to strain rate effect. 


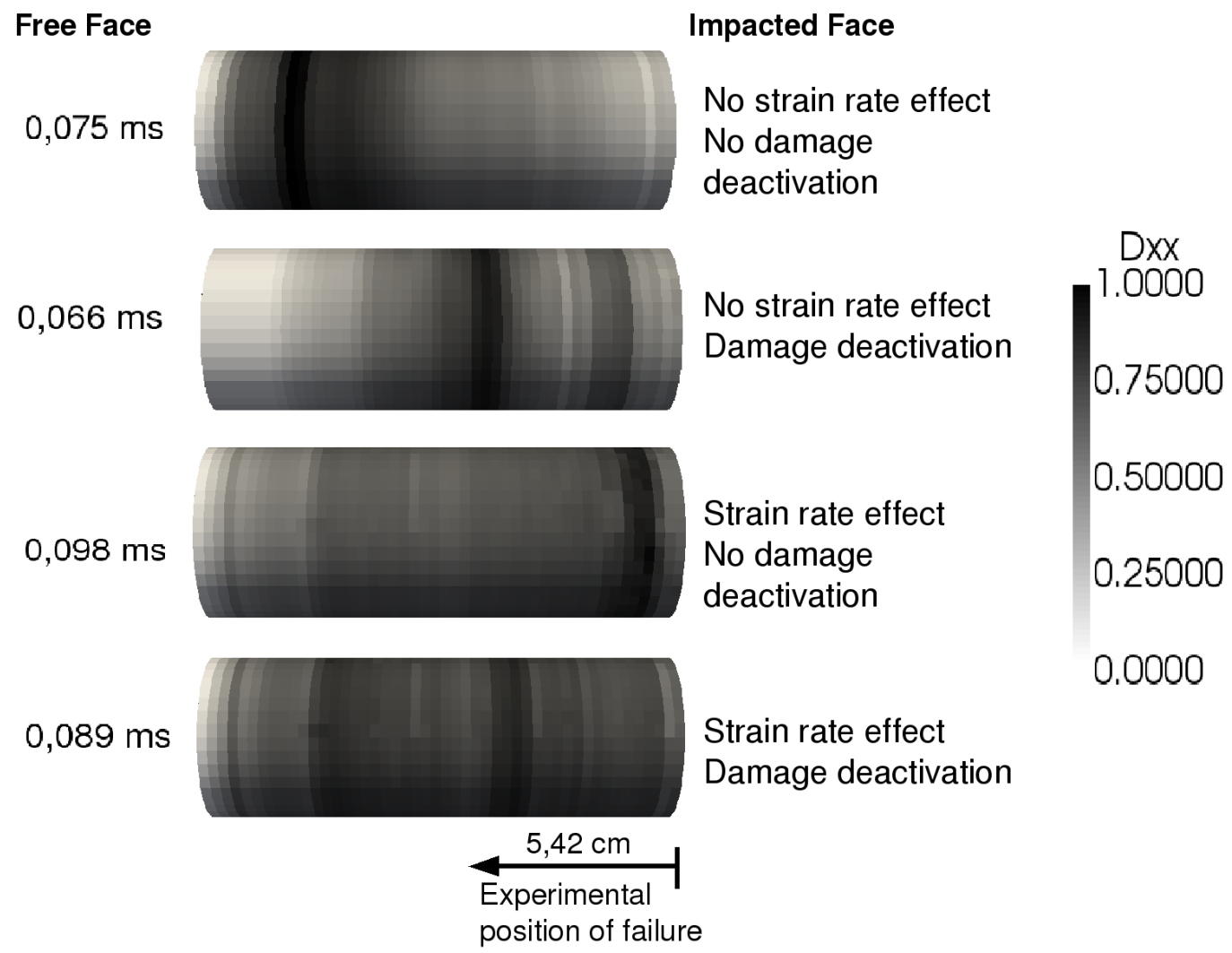

Figure 11: Damage map $D_{x x}$ for the differents version of the anisotropic damage

\subsection{Regularization in dynamics}

Delay-damage and non-local computations are efficient tools do deal with localization issues, but each of these model enhancements has its own domain of validity in terms of strain rate. In this section, one quantifies for which strain rate range it is relevant to apply these localization limiters

The micro-deffects closure modeling - by means of the active damage concept - has no influence on the efficiency of the regularization. Therefore, the results presented next without damage deactivation are relevant to qualify the quality of the regularization. Four models are compared (without damage deactivation then):

- initial local model, no visco-damage (i.e. without regularization case),

- local delay-damage model with criterion function expressed in terms of $\operatorname{tr} \boldsymbol{D}$,

- initial non-local model, no visco-damage,

- non-local delay-damage model which combines the two previous models, the delay-damage law reading:

$$
\mathcal{H}(\operatorname{tr} \boldsymbol{\varepsilon}) \operatorname{tr} \dot{\boldsymbol{D}}=\dot{D}_{\infty}\left[1-\exp \left(-b\left(g\left(\hat{\boldsymbol{\varepsilon}}^{n l}\right)-\operatorname{tr} \boldsymbol{D}\right)\right)\right]
$$

The parameters are still those of table 1, i.e. the delay-damage parameters are those representing the experimental strain rate effect and are therefore not chosen for regularization purpose.

In order to study the regularization, two results are observed: $D_{x x}$ damage fields and the evolution of the ejection velocity. If damage is localized in one (or a few) element zone, whatever the size of the elements, regularization is not efficient. What is called ejection velocity corresponds to the evolution of axial velocity $v_{x}$ of the center of the free 
face. When failure occurs, the part of the specimen located on the left of the crack is ejected. The ejection velocity depends on where and when this happens, therefore it is a good indicator of the mesh dependency.

One compares the results for 3 meshes (coarse 3300 DOF, medium 6468 DOF et fine 12804 DOF, cf. Fig. 12). To illustrate the need of regularization, a computation with the same meshes and the initial model (without non-local regularization nor delay-damage) is performed first (results in Fig. 13 and Fig. 14).

Finally two different impact velocities are compared in order to establish at which strain rates the regularization methods are efficient. The case called "fast impact" corresponds to the experimental case described above. The loading for the case called "slow impact", described in paragraph 6.5, consists in applying the same maximum displacement but in a 4 times longer time. The specimen geometry has to be adapted.

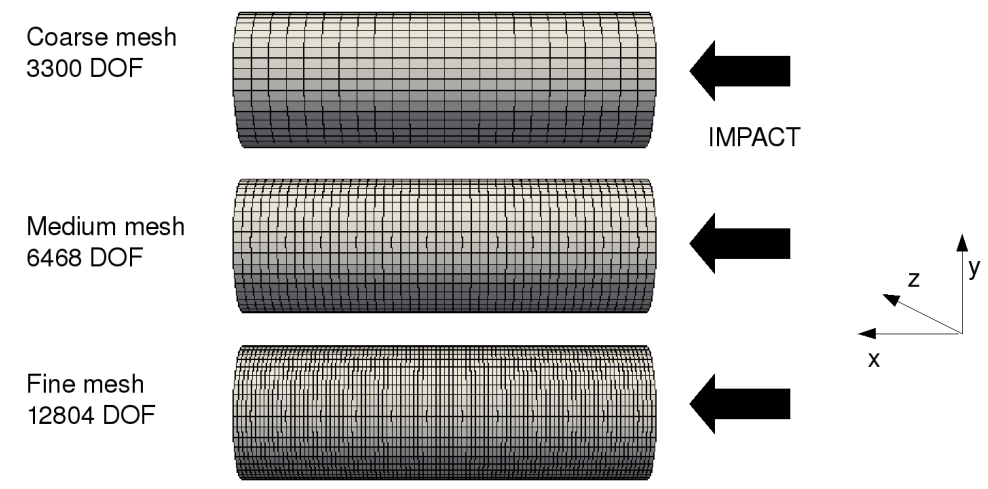

Figure 12: 3 Meshes used for fast impact

\subsection{Fast impact}

\subsubsection{Without Regularization}

Without regularization, damage is classically localized in a thin area (Fig. 13), which reproduces well the kind of failure observed. If one looks at the ejection velocity (Fig. 14), the result is clearly mesh dependent. The three curves obtained for the three meshes are not superimposed anymore from the time at which strain and damage localization occurs $(\mathrm{t} \simeq 0.75 \mathrm{~ms})$. This is typical from a non-regularized computation. The results are not reliable since they depend on the discretization chosen.

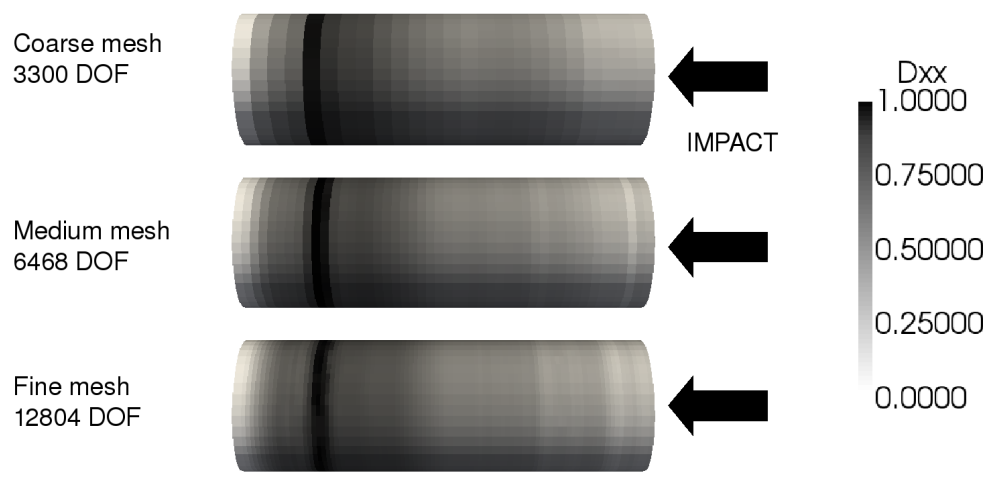

Figure 13: Damage maps $D_{x x}$ without regularization - fast impact 


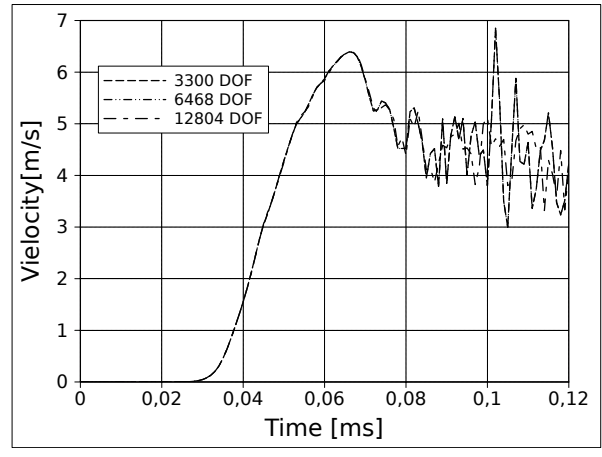

(a) Complete evolution

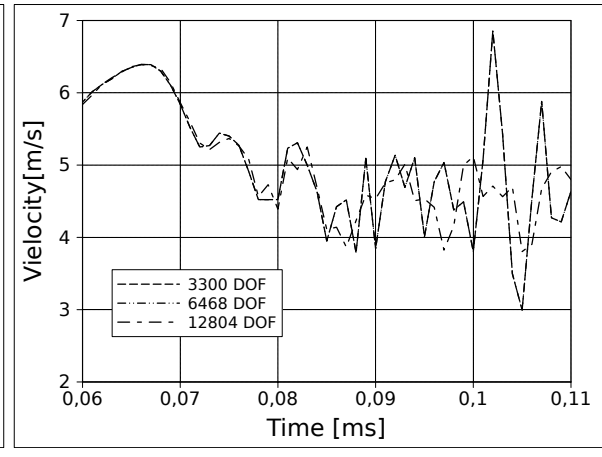

(b) Detail

Figure 14: Ejection velocity without regularization - fast impact

\subsubsection{Delay-damage regularization}

In that case, the thickness of the damaged area is not limited to a low number of elements. For the coarser mesh, the critical damage is reached in 2 rows of elements (Fig. 15). The velocity evolution (Fig. 16) shows less important differences between the results for the different meshes than without regularization. We can consider, given the weak mesh dependency, that the solution is regularized in that case (theoretical results with delay damage can be found in [39]). For the strain rate range considered (of the order of magnitude of $100 \mathrm{~s}^{-1}$ ), delay-damage is an efficient regularization tool.

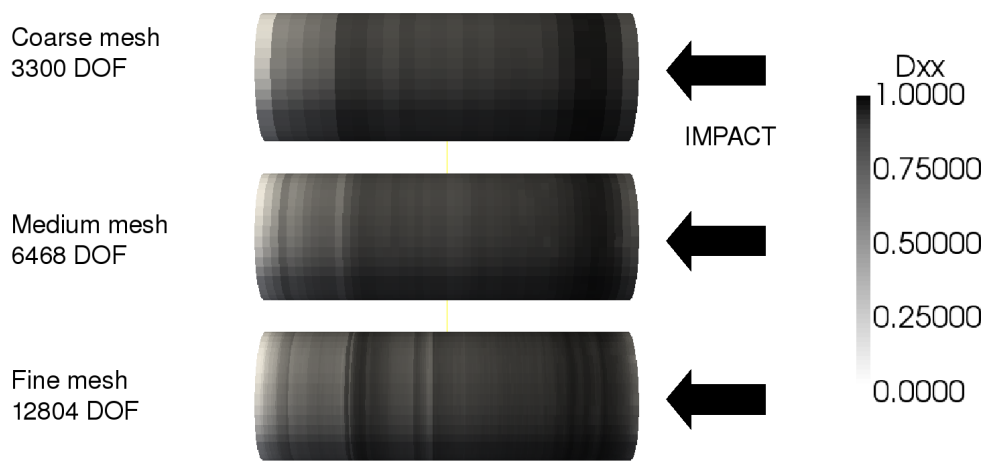

Figure 15: Damage maps $D_{x x}$ with delay-damage regularization - fast impact

\subsubsection{Non-local regularization}

The internal length $l_{c}$ is taken equal to $1.5 \mathrm{~cm}$ for the three meshes, which corresponds to 3 elements (in the longitudinal direction) for the coarse mesh, 6 for the medium one and 12 for the fine one.

The 3 damage maps (Fig. 17) exhibit a larger damage zone that previously (about $3 \mathrm{~cm}$ ), which tends to decrease with the mesh size. It looks as if non-local regularization in dynamics spreads damage in a too important zone. The association of non-local enhancement, which averages the equivalent strain in a defined area (therefore make non-local damage evolution), with fast wave propagation is not able to describe a localized failure. Nevertheless, the solution is perfectly regularized, as seen on the ejection velocity curves (fig 18), in which the results exhibit absolutely no mesh dependency. 


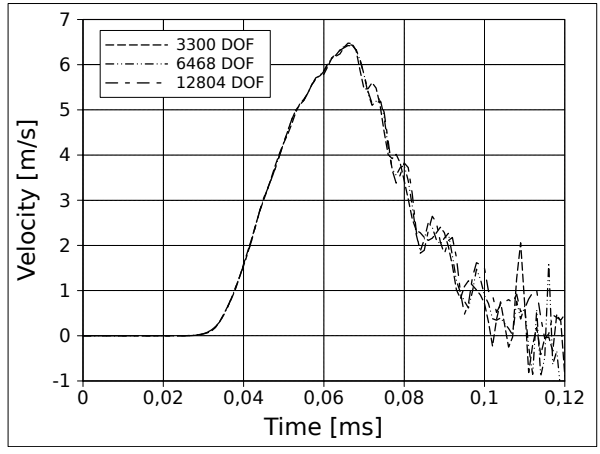

(a) Complete evolution

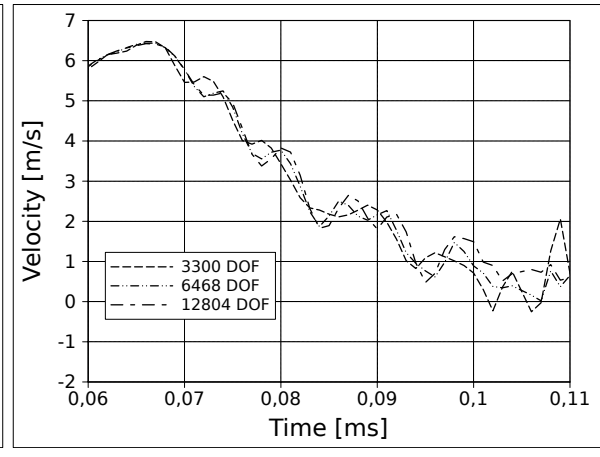

(b) Detail

Figure 16: Ejection velocity with delay-damage regularization - fast impact

Coarse mesh 3300 DOF
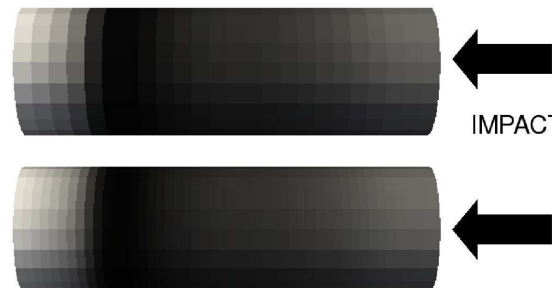

IMPACT

Medium mesh 6468 DOF

Fine mesh 12804 DOF

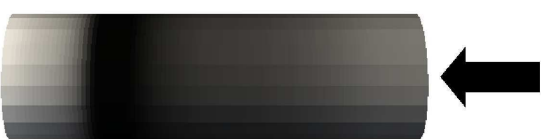

$\mathrm{Dxx}$

1.0000

0.75000

0.50000

0.25000

0.0000

Figure 17: Damage maps $D_{x x}$ with non-local regularization - fast impact

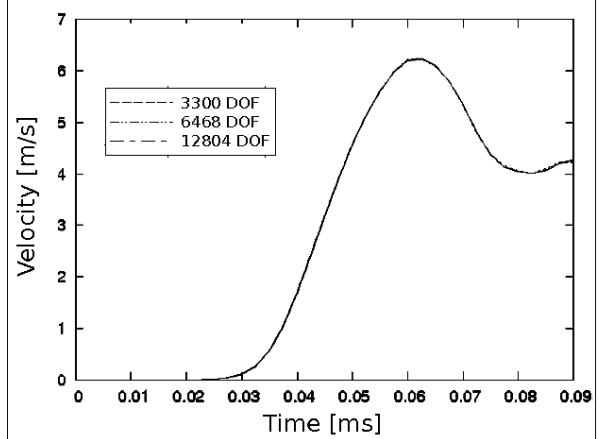

(a) Complete evolution

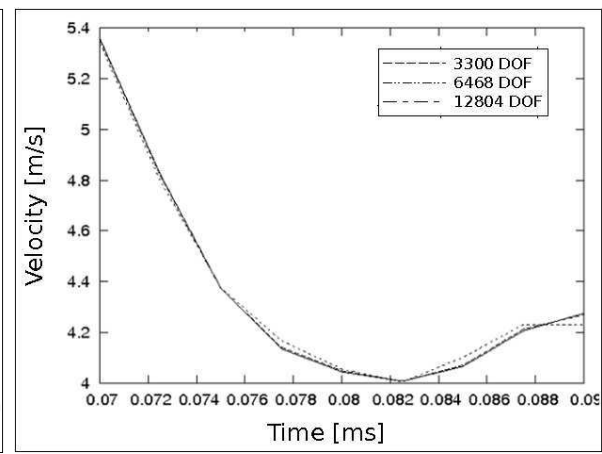

(b) Detail

Figure 18: Ejection velocity with delay-damage and non-local regularization - fast impact

\subsubsection{Delay-damage and non-local regularization}

The idea of combining delay-damage and non-local regularization aims at taking advantage of both: good regularization thanks to non-local formulation and representation of the experimental strain rate effect with visco-damage. But, as seen in figure 19, this choice seems not appropriate as it leads to an non-homogeneous damage state inside the 
specimen. The edge effect of non-local averaging is found very high around the lateral face, so that a too low damage level is obtained far from the center of the spall plane (in top and bottom areas of cross section view). Furthermore, failure occurs close to the (left) free face of the specimen, as if the strain rate effect were not represented. However, as shown by both the damage maps and the velocity evolution (Fig. 20), the solution is perfectly regularized.

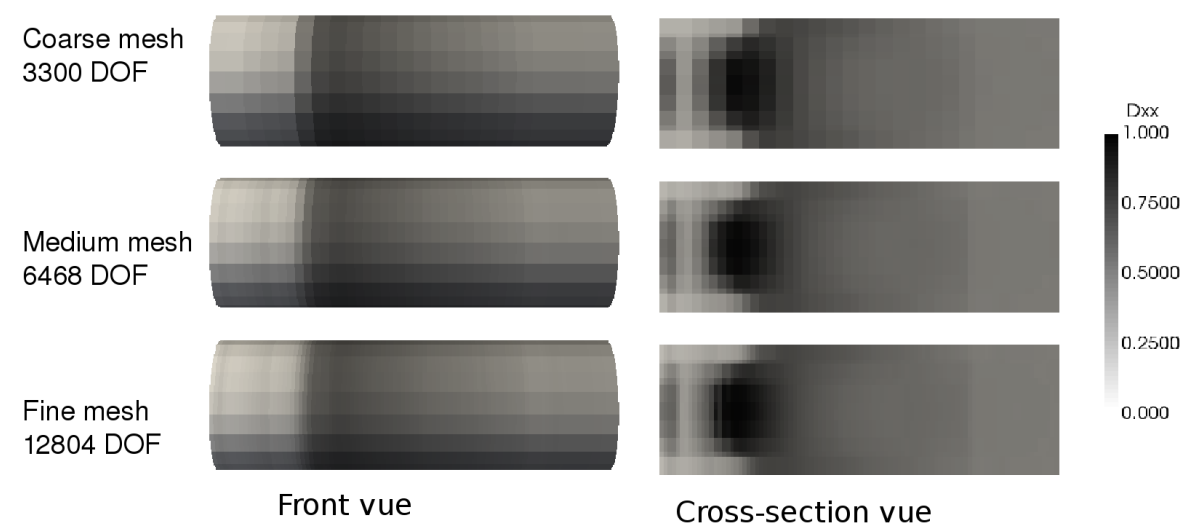

Figure 19: Damage maps $D_{x x}$ with non-local regularization - fast impact

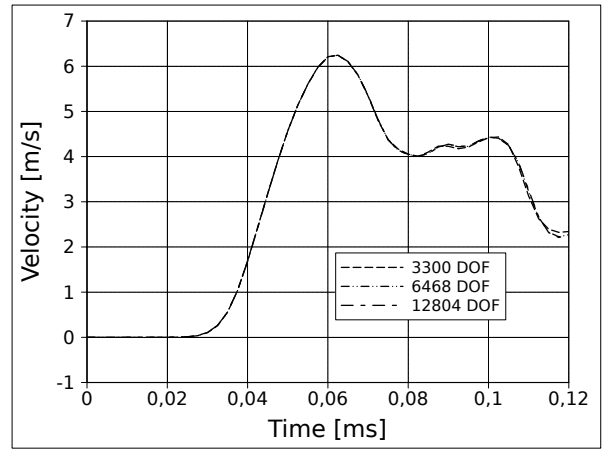

(a) Complete evolution

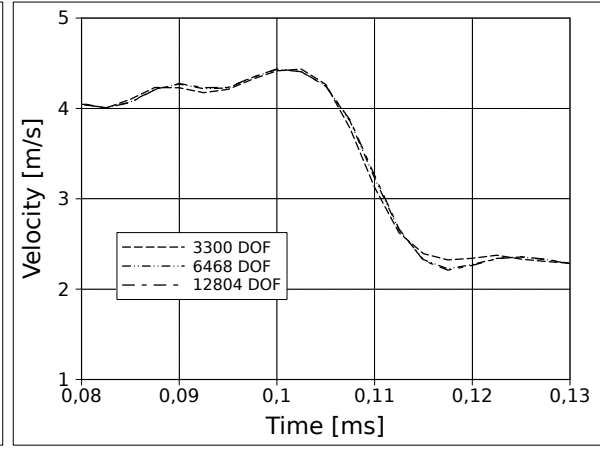

(b) Detail

Figure 20: Ejection velocity with delay-damage and non-local regularizations - fast impact

\subsection{Slow impact}

In the previous paragraph, it has been established that delay-damage regularization was the best adaptated for high-rate dynamics. One aims here at studying the case of slower dynamics, where the strain rate effect is lower or even negligible. Therefore, the same impact computations are performed but with a slower loading. The maximum displacement imposed to the right face specimen remains the same, but the loading duration is multiplied by 4 . If the bar length is kept unchanged, the incident wave starts to reflect on the left free face before the maximum displacement is reached. rom this results that the stress state is a complex superimposition on tension and compression waves which does not lead to failure. For that reason, the geometry considered next is chosen longer $(96 \mathrm{~cm}$, i.e. 4 times previous length) but keeps the same diameter $(2 \mathrm{~cm})$. Three different meshes, with the same number of degrees of freedom as before, are compared (Fig. 21). For that example, the average strain rate is around $3 \mathrm{~s}^{-1}$. 


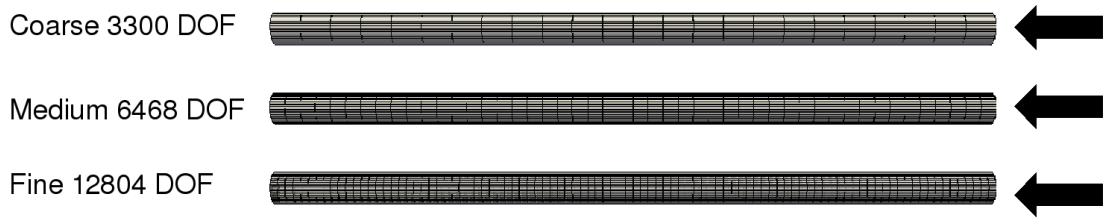

Impact

Figure 21: 3 meshes for slow impact

\subsubsection{Without Regularization}

Without regularization, damage is localized in a thin band, which decreases when the mesh is refined (Fig. 22).

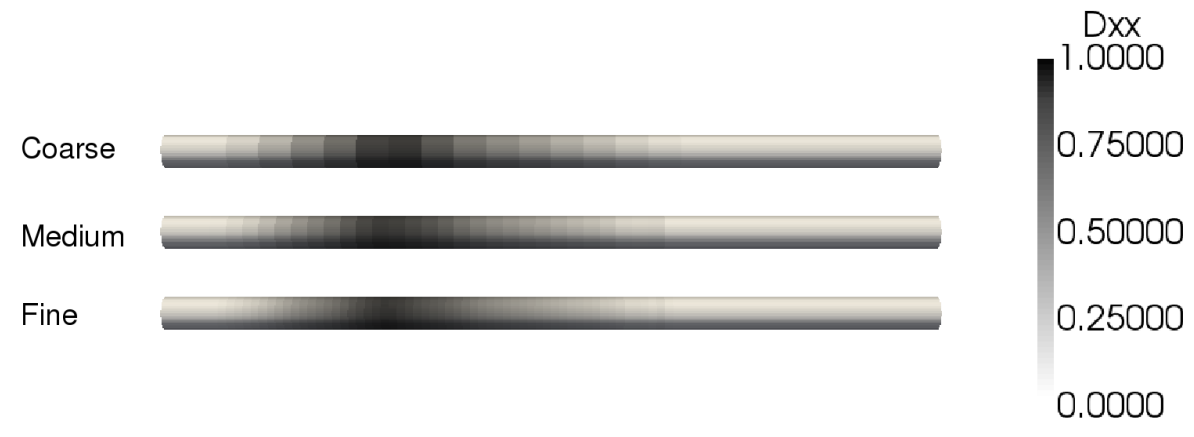

Figure 22: Damage maps $D_{x x}$ without regularization - slow impact

When localization occurs (around $\mathrm{t}=0,4 \mathrm{~ms}$ ), ejection velocity curves (Fig. 23) stop to superimpose and exhibit large differences between them (mesh dependency).

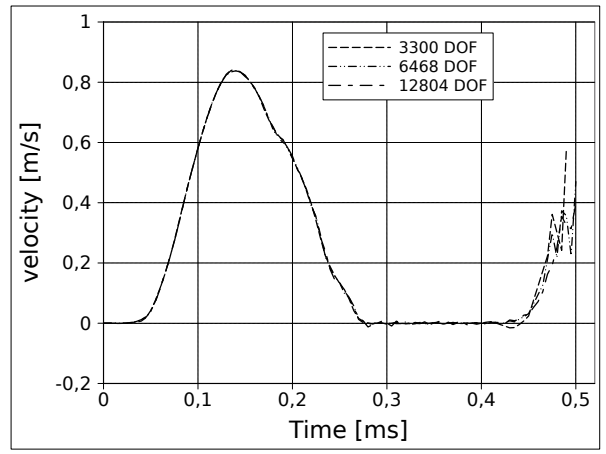

(a) Complete evolution

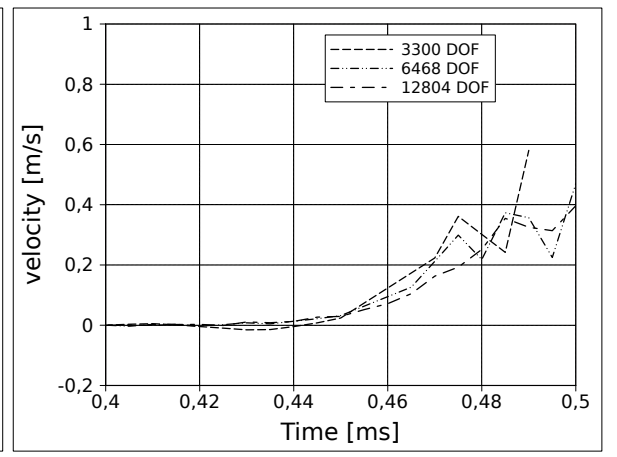

(b) Detail

Figure 23: Ejection velocity without regularization - slow impact

\subsubsection{Delay-damage regularization}

With delay-damage (Fig. 24), damage maps exhibit mesh dependency as in the no regularization at all case. Two different localization bands are obtained, even three with the finer mesh. 


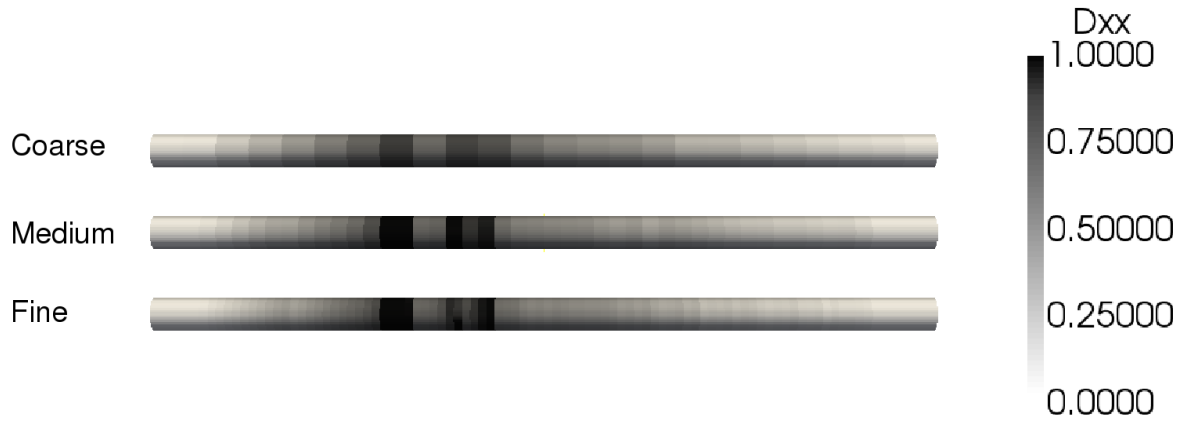

Figure 24: Damage maps $D_{x x}$ with delay-damage regularization - slow impact

Ejection velocity evolution curves (Fig. 25) confirm the fact that for low-rate dynamics delay-damage cannot be considered as a regularization method if the delay-damage parameters are identified from the material strain rate effect. In the case studied, to model the strain rate effect has a small influence on the result (the localization band is more distant from the (left) free face where reflection takes place, which means that the concrete strength is higher), but not on the mesh dependency.

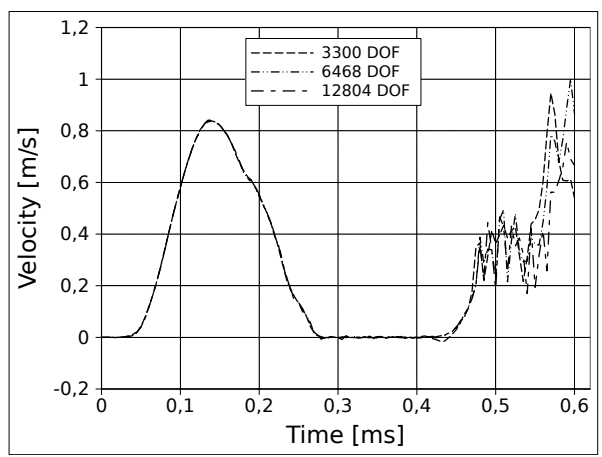

(a) Complete evolution

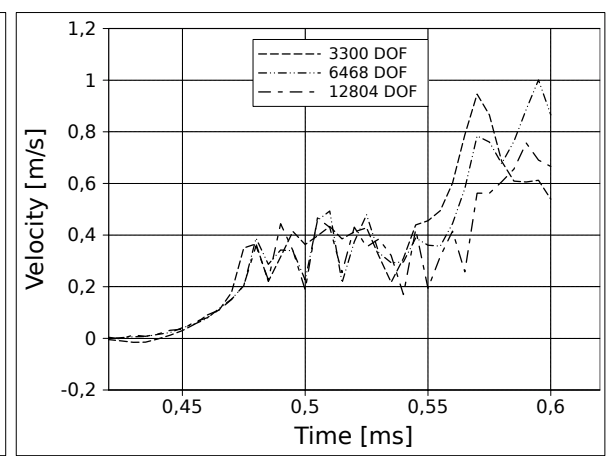

(b) Detail

Figure 25: Ejection velocity with delay-damage regularization - slow impact

\subsubsection{Non-local regularization}

As expected and as for fast dynamics, non-local regularization leads to a perfectly regularized solution in terms of damage maps (Fig. 26) as well as for velocity evolutions (Fig. 27). The localization band remains however quite large in that case.

\subsubsection{Delay-damage and non-local regularization}

Delay-damage associated with non-local enhancement leads to a solution which does not present the same regularization quality as single non-local enhancement or single delay-damage, separately. The damage maps present a variable number of localization bands (Fig. 28) and the differences observed between the velocity evolutions are significant (Fig. 29). 


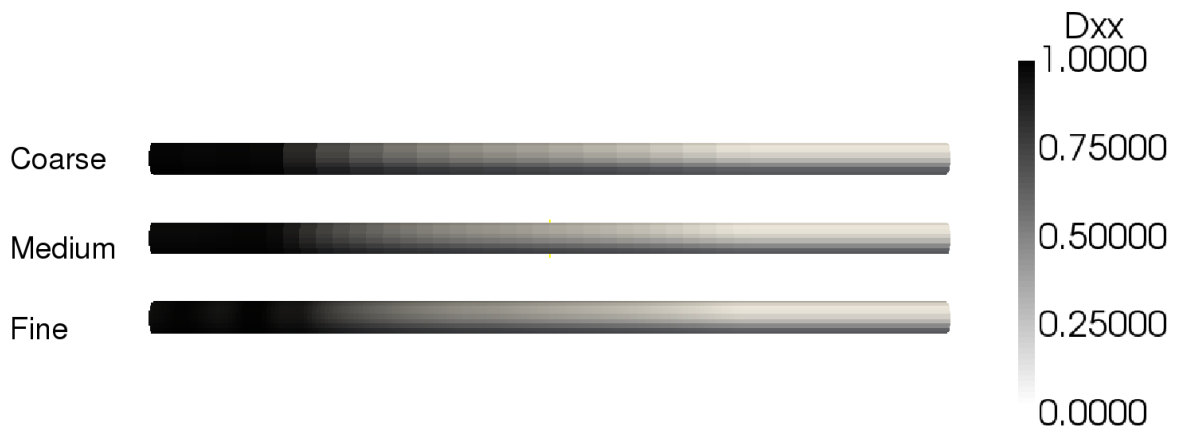

Figure 26: Damage maps $D_{x x}$ with non-local regularization - slow impact

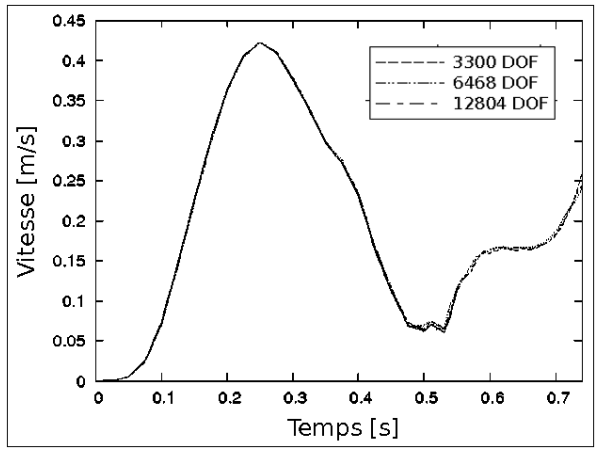

(a) Complete evolution

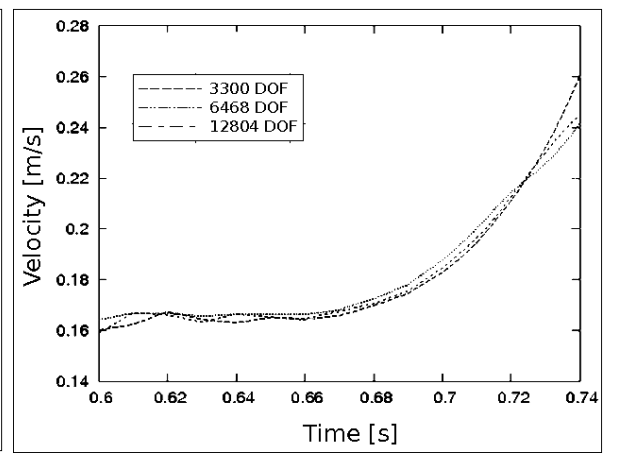

(b) Detail

Figure 27: Ejection velocity with non-local regularizations - slow impact

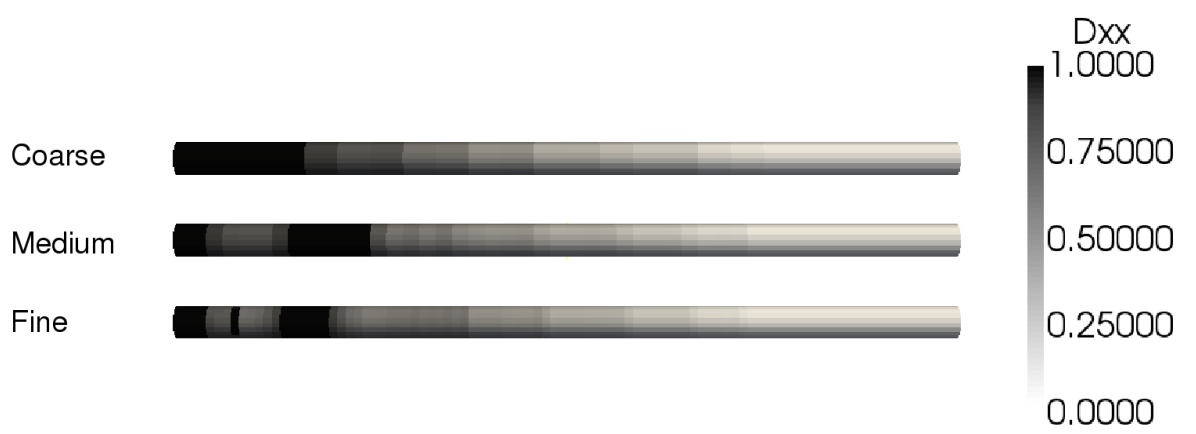

Figure 28: Damage maps $D_{x x}$ with delay-damage and non-local regularizations - slow impact

\section{Conclusion}

A 3D anisotropic visco/delay-damage model for concrete in dynamics is developed in a modular formulation. Each modeling elementary block, the delay-damage one, the active damage one, the nonlocal enhancement, can be activated or not. Important feature, the material parameters already identified are kept unchanged when switching from one model version to another one. The numerical scheme proposed is robust and quite simple (as Euler backward scheme is used and as there is no need for iterations at Gauss point level). 


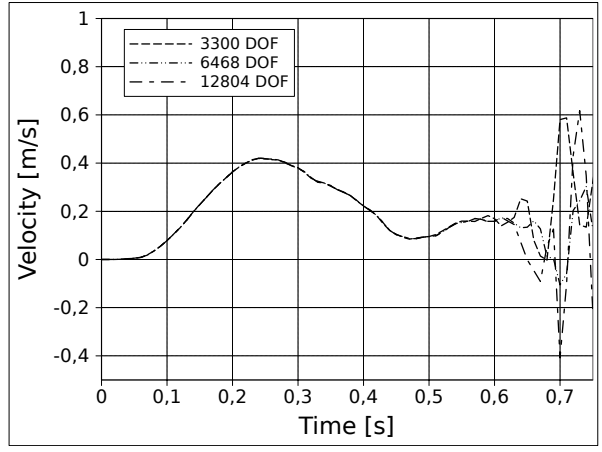

(a) Complete evolution

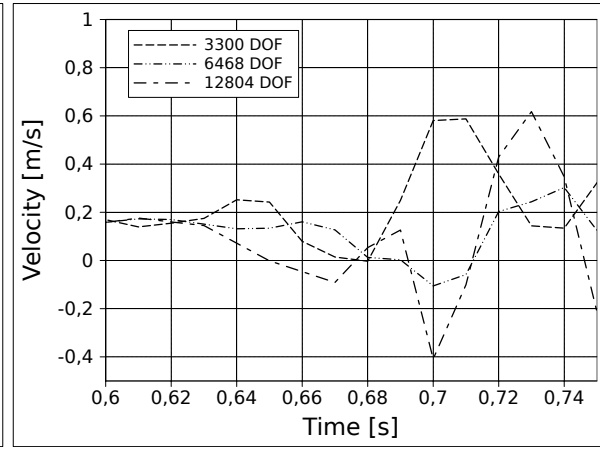

(b) Detail

Figure 29: Ejection velocity with delay-damage and non-local regularizations - slow impact

Thanks to the introduction of Heaviside term $\mathcal{H}(\operatorname{tr} \boldsymbol{\varepsilon})$ in delay-damage evolution law, the model represents the strain rate effect observed in tension while the apparent strength increase in compression is simply obtained by considering inertia forces.

Active damage has been defined and introduced in the so-called anisotropic delay-active damage model. It has proven to be a key concept to deal with alternated loading (most usual in wave propagation and reflection cases). It is introduced in both the damage criterion function and the delay-damage evolution law, for both quasi-statics and dynamics applications. A main conclusion of present work is that active damage should not be considered as an optional elementary brick of the model but should be included in the basic constitutive equations of the anisotropic damage model.

Regularization properties have been studied from 3D computations of dynamics tension tests with Hopkinson bars for different versions of the anisotropic model. The corresponding conclusions are:

- Classically, the anisotropic delay-damage model proves its ability to regularize the Finite Element solution if the strain rate is large enough to have a significant effect on the stress-strain response and on the apparent strength increase. Less classically and result specific to concrete, the parameters identified to represent the material strain rate effect are efficient for high-rate dynamics regularization purpose.

- With the same delay-damage model (and parameters), the low-rate dynamics computations have not been regularized.

- Non-local enhancement, an efficient regularization method in quasi-statics as well as in dynamics, has not given satisfactory results for the location of the failure plane (even when combined with delay-damage). The nonlocal averaging seems to interact too much with wave propagation and changes much the location of the failure plane. Edges effect have also been found too pronounced.

Both non-local enhancement and delay-damage have then their (separate) domain of validity in terms of strain rates. A model valid for any strain rate will combine both, but will make the non-local characteristic length a decreasing function of the strain rate, setting for example

$$
l_{c}=l_{c}(\dot{\varepsilon})=l_{c}^{0} \exp \left[-\left(\frac{\dot{\varepsilon}}{\dot{\varepsilon}^{\star}}\right)^{n}\right]
$$

with $l_{c}^{0}$ the usual quasi-static characteristic length, $\dot{\varepsilon}^{\star}$ and $n$ additional material parameters. At low strain rates $l_{c}(\dot{\varepsilon}) \approx$ $l_{c}^{0}=C s t$ and the model recovers the classical non-local model, when at high strain rates $l_{c} \rightarrow 0$ and the model tends toward the local delay-damage model. 


\section{References}

[1] J. Mazars, Y. Berthaud and S. Ramtani. The unilateral behavior of damage concrete. Engng Fract. Mech. 1990; 35:629-35.

[2] J.L. Chaboche, P.M. Lesne, J.F. Maire, Phenomenological Damage Mechanics of Brittle Materials with Description of the Unilateral Damage Effect, in: Z.P. Bazant et al. (Eds.), Fracture and Damage in Quasibrittle Structures, E.\& F.N. Spon Pubs, London, pp. 75-84, 1994.

[3] K. Willamand and E. Warnke. Constitutive model for triaxial behaviour of concrete, Proc. Concrete and Structures Subjected to Triaxial Stresses, Int. Ass. for Bridge and Structural Engineering, Zurich. 1975: 1-30.

[4] M. Ortiz. A constitutive theory for the inelastic behavior of concrete, Mech. Mater. 1985;4:67-93.

[5] G. Voyiadjis and T. Abu-Lebdeh. ,Plasticity model for concrete using the bouding surface model. Int. J. Plast. 1994;10:1-21.

[6] P.H. Feenstra and R.de Borst. A composite plasticity model for concrete. Int. J. Solids Struct. 1996;33(5):707-730.

[7] J. Lemaitre, J.L. Chaboche, A. Benallal, R. Desmorat, Mécanique des matériaux solides, 3rd edition, Dundod Paris, 2009.

[8] J.C. Simo and J.W. Ju, International Journal of Solids and Structures, 23(7), pp. 821-840, 1987.

[9] J.W. Ju. On energy-based coupled elastoplastic damage theories: constitutive modeling and computational aspects Int. J. Solids structure $1989 ; 25(7)$.

[10] F. Gatuingt and G. Pijaudier-Cabot. Gurson's plasticity coupled to damage as a CAP Model for concrete compaction in dynamics, in Constitutive Modelling of Geomaterials, CRC Press. 2003:12-24.

[11] J. Lemaitre and R. Desmorat. Engineering damage mechanics: ductile, creep, fatigue and brittle failures Springer 2005.

[12] L. Jason, A. Huerta, G. Pijaudier-Cabot and S. Ghavamian. An elastic plastic damage formulation for concrete: Application to elementary tests and comparison with an isotropic damage model Comput. Meth. Appl. Mech. Engng 2006;195(54):7077-92.

[13] R. Desmorat, F. Ragueneau, H. Pham, Continuum Damage Mechanics for hysteresis and fatigue of quasi-brittle materials and structures, International Journal of Numerical and Analytical Methods for Geomaterials, vol. 31, p. 307-329, 2007.

[14] M. Matallah and C. La Borderie, Inelasticity-damage-based model for numerical modeling of concrete cracking, Engng Fract. Mech., 76(8), pp. 163-79, 2009.

[15] J. Mazars. Application de la mécanique de l'endommagement au comportement non linéaire et à la rupture du béton de structure. Doctorat d'état de l'université de Paris VI 1984

[16] W. Suaris and S.P. Shah. Test Methods for Impact Resistance of Fiber Reinforced Concrete Am. Concrete Inst.: SP-81:247-66, 1984.

[17] J.L. Chaboche. The continuous damage mechanics. A tool to describe phenomena before crack initiation, Nucl. Engng Des. 1979.

[18] J.-P. Cordebois and J.P. Sidoroff. Endommagement anisotrope en élasticité et plasticité J.M.T.A, Numéro spécial 1982:45-60.

[19] P. Ladeveze. On an anisotropic damage theory, In: Proc. CNRS Int. Coll 351 Villars-de-Lans, Failure crieria of structured media. 1983.

[20] S. Fichant, C. La Borderie, G. Pijaudier-Cabot, Isotropic and Anisotropic Descriptions of Damage in Concrete Structures, Int. J. of Mechanics of Cohesive-Frictional Materials 5 (1998) 339-359.

[21] J. Mazars, Y. Berthaud and S. Ramtani. The unilateral behaviour of damaged concrete Engng Fract. Mech. 1990;35(4):629-35.

[22] E. Papa and A. Talierco. Anisotropic damage model for the multi-axial static and fatigue behavior of plain concrete. Engng Fract. Mech. 1996;55:163-79.

[23] S. Murakami and K. Kamiya. Constitutive and damage evolution equations of elastic-brittle materials based on thermodynamics. Int. J. Mech. Sci., 39(4):459-73, 1996.

[24] A. Dragon and D. Halm. A model of anisotropic damage by mesocrack growth; unilateral effect, Int. J. Damage Mech., 5(4):384-402, 1996.

[25] J. Lemaitre, R. Desmorat and M. Sauzay. Anisotropic damage law of evolution. European J. Mechanics A/Solids 2000;19:187-208.

[26] P. Badel, V. Godard, J.-B. Leblond, Application of some anisotropic damage model to the prediction of the failure of some complex industrial concrete structure, Int. J. Solids and Structures, 44, pp. 5848-5874, 2007.

[27] R. Desmorat. Modèle d'endommagement anisotrope avec forte dissymétrie traction/compression. (RF)2B - 5es Journées Scientifiques du Regroupement Francophone pour la Recherche et la Formation sur le Béton 2004

[28] R. Desmorat, F. Gatuingt and F. Ragueneau. Nonlocal anisotropic damage model and related computational aspects for quasi-brittle materials. Engng Fract. Mech. 2007;74(10):1539-60.

[29] R. Desmorat, F. Gatuingt, F. Ragueneau, Non standard thermodynamics framework for robust computations with induced anisotropic damage, International Journal of Damage Mechanics, 19(1), pp. 53-73, 2010.

[30] Taylor, L, M., Chen, E. P. and Kuszmaul, J. S., Microcrack-lnduced Damage Accumulation in Brittle Rock Under Dynamlc Loading, Computer Methods in Applied Mechanics and Engineering, 55(3), pp. 301-320, 1986.

[31] P.H. Bischoff and S.H. Perry, Compressive behaviour of concrete at high strain rates, Mater. Struct., 24(6):425-50, 1991.

[32] A. Brara, J. Klepaczko, J. and L. Kruszka. Tensile testing and modeling of concrete under high loading rates. In Brandt: Li and Marshall (Eds.), Brittle Matrix Composites 5 (BMC5), Warsaw, Poland 1997.

[33] A. Brara and JR. Klepaczko. Nouveau dispositif exprimental d'étude de la rupture par écaillage du béton. Communication-Congres GEO, Aussois, France, 1997.

[34] A. Brara, F. Camborde, JR. Klepaczko and C. Mariotti. Experimental and numerical study of concrete at high strain rates in tension. Mech. Mater., 33(1):33-45, 2001.

[35] J. Klepaczko and J. Brara, An experimental method for dynamic tensile testing of concrete by spalling, Int. J. Impact Engng, $25(4), 2001$.

[36] A. Brara J. Klepasczko. Experimental characterization of concrete in dynamic tension. Mech. Mater. 2006;38(3):253-67.

[37] C. Pontiroli, Comportement au souffle des structures en béton armé. PhD Ecole Normale Supérieure de Cachan, France, 1995.

[38] J.-F. Dubé, G. Pijaudier-Cabot and C. La Borderie, Rate dependent damage model for concrete in dynamics, Journal of Engineerings Mechanics, 122(10), pp. 939-947, 1996.

[39] O. Allix, J.F. Deü. Delayed-damage modelling for fracture prediction of laminated composites under dynamic loading. Engng Trans 1997;45:29-46.

[40] J. Sercombe, F.J. Ulm and F. Toutlemonde. Viscous hardening plasticity for concrete in high-rate dynamics, J. Engng Mech. 1998;124:1050-7.

[41] F. Gatuingt and G. Pijaudier-Cabot. Coupled damage and plasticity modelling in transient dynamic analysis of concrete, Int. J. Numer. Anal. Meth. Geomech., 26:1-24, 2002. 
[42] R.R. Pedersen, A. Simone and L.J. Sluys. An analysis of dynamic fracture in concrete with a continuum visco-elastic visco-plastic damage model Engng Fract. Mech. 2008;75:3782-805.

[43] U. Haussler-Combe and M. Kitzig: Modeling of concrete behavior under high strain rates with initially retarded damage, International Journal of Impact Engineering, 36, 1106-1115, 2009.

[44] C. La Borderie , Y. Berthaud and G. Pijaudier-Cabot. Crack closure effect in continuum damage mechanics: numerical implementation. In: Proceedings of the Second international Conference on 'Computer aided analysis and design of concrete structures'. Zell am See, Austria, 4-6 April, 1990:975-86.

[45] F. Ragueneau, C. La Borderie, J. Mazars, Damage model for concrete like materials coupling cracking and friction - Contribution towards structural damping: first uniaxial application, Mechanics Cohesive Frictional Materials, 5, pp. 607-625, 2000.

[46] A. Souid, A. Delaplace, F. Ragueneau and R. Desmorat, Pseudodynamic testing and nonlinear substructuring of damaging structures under earthquake loading, Engng Struct., 32(5):1102-10, 2009.

[47] G. Pijaudier-Cabot, Z.P. Bazant, Nonlocal Damage Theory, J. Engng Mech., 113(10), pp. 1512-1533, 1987.

[48] R. Peerlings. Enhanced damage modelling for fracture and fatigue. PhD thesis. TU Eindhoven - The Netherlands, 1999.

[49] R. H. J. Peerlings, M. G. D. Geers, R. de Borst and W. A. M. Brekelmans, A critical comparison of nonlocal and gradient-enhanced softening continua, Int. J. Solids \& Structures, 38, pp. 7723-7746, 2001.

[50] J.H.P. de Vree, W.A.M. Brekelmans and M.A.J. van Gils. Comparison of nonlocal approaches in continuum damage mechanics, Comput. Struct., 55(4), pp. 581-8, 1995.

[51] L. Agardh, L. Laine, 3D FE-simulation of high-velocity fragment perforation of reinforced concrete slabs, International Journal of Impact Engineering, 22, pp. 911-922, 1999.

[52] E. P. Chen, Non-local effects on dynamic damage accumulation in brittle solids, International Journal for Numerical and Analytical Methods in Geomechanics 23(1), pp. 1-21, 1999.

[53] Y. Lu, K. Xu, Modelling of dynamic behaviour of concrete materials under blast loading, International Journal of Solids and Structures, 41(1), pp. 131-143, 2004.

[54] G. Z. Voyiadjis, R. K. Abu Al-Rub and A. N. Palazotto, Thermodynamic framework for coupling of non-local viscoplasticity and non-local anisotropic viscodamage for dynamic localization problems using gradient theory, International Journal of Plasticity, 20(6), pp. 981-1038, 2004.

[55] A. Needleman. Material rate dependance and mesh sensitivity in localization problems. Comput. Meth. Appl. Mech. Engng, 67:69-85, 1988.

[56] P. Ladevèze, On a damage mechanics approach. In: D Baptiste, Editor, Proc. 4th Int. Mecamat Seminar on Mechanics and Mechanisms of Damage in Composites and Multi-materials, Mechanical Engineering Publication (1991), pp. 119-141.

[57] J. Eibl, Strain-Rate-Sensitive Constitutive Law for Concrete, Journal of Engineering Mechanics, 125(12), pp. 1411-1420, 1999.

[58] G. Hervé, F. Gatuingt, A. Ibrahimbegovic, On numerical implementation of a coupled rate dependent damage-plasticity constitutive model for concrete in application to high-rate dynamics, Engineering Computations 22(5/6), pp. 583-604, 2005.

[59] Ragueneau F., Desmorat R. and Gatuingt F., Anisotropic damage modelling of biaxial behaviour and rupture of concrete structures, Computers and Concrete, 5(4), pp. 417-434, 2008.

[60] E.C. Aifantis. On the microstructural origin of certain inelastic models. J. Engng Mater. Techol. 1984;106(4):326-30.

[61] W. F. Brace and A.H. Jones. Comparison of uniaxial deformation in shock and static loading of three rocks, J. Geophys. 1 Res., 1971;76(20):4913-21.

[62] Q.M. Li and H. Meng. About the dynamic strength enhancement of concrete-like materials in a split Hopkinson pressure bar test, Int. J. Solids Struct., 40:343-60, 2003.

[63] F. Ragueneau, F. Gatuingt, Inelastic behavior modelling of concrete in low and high strain rate dynamics. Computers \& Structures, 8, pp. $1287-1300,2003$

[64] H.B. Kupfer, H.K. Hilsdorf and H. Rusch. Behavior of concrete under biaxial stresses Am. Concr. Inst. J., 1979;66(8):343-60.

[65] P. Rossi, J.G.M. Van Mier, F. Toutlemonde, F. Le Maou and C. Boulay. Effect of loading rate on the strength of concrete subjected to uniaxial tension. Mater. Struct., 1994;27:260-4.

[66] D.R.J. Owen and F. Damjanic. Viscoplastic analysis of solids: stability considerations. Recent advances in non-linear computational mechanics, Eds E. Hinton 1982:225-53.

[67] F. Gatuingt, R. Desmorat, M. Chambart, D. Combescure, D. Guilbaud. Anisotropic 3D delay-damage model to stimulate concrete structures. Revue Européenne de mécanique numérique 2008;17:740-60.

[68] M. Chambart. Endommagement anisotrope et comportement dynamique des structures en béton armé jusqu'à la ruine. PhD Ecole Normale Supérieure de Cachan, 2009.

[69] C. Denoual and F. Hild. A damage model for the dynamic fragmentation of brittle solids Comput. Meth. Appl. Mech. Engng 2000;183:24758.

[70] R. Desmorat, Positivity of intrinsic dissipation of a class of nonstandard anisotropic damage models, C.R. Mcanique, vol. 334, p. 587-592, 2006.

[71] A.C. Eringen, Microcontinuum field theories, Springer, New York, 1999.

[72] R. de Borst, L.J. Sluys, Localisation in a Cosserat continuum under static and dynamic loading conditions, Comp. Methods Appl. Mech. Eng., 90, pp. 805-827, 1991.

[73] S. Forest, Micromorphic approach for gradient elasticity, viscoplasticity, and damage, J. Engng Mech., 135(3), pp. 117-131, 2009.

[74] T.J. Massart, R.H.J. Peerlings and M.G.D. Geers, Mesoscopic modeling of failure and damage-induced anisotropy in brick masonry, Eur. J. Mech. A/Solids, 23,(5), pp. 719-735, 2004.

[75] Z.P. Bazant and M. Jirasek, Nonlocal Integral Formulations of Plasticity and Damage: Survey of Progress, J. Engrg. Mech., 128(11), pp. 1119-1149, 2002 